\title{
Holonomy and Projective Equivalence in 4-Dimensional Lorentz Manifolds ${ }^{\star}$
}

\author{
Graham S. HALL ${ }^{\dagger}$ and David P. LONIE ${ }^{\ddagger}$ \\ $\dagger$ Department of Mathematical Sciences, University of Aberdeen, \\ Meston Building, Aberdeen, AB24 3UE, Scotland, UK \\ E-mail: g.hall@abdn.ac.uk \\ $\ddagger 108$ e Anderson Drive, Aberdeen, AB15 6BW, Scotland, UK \\ E-mail:DLonie@aol.com
}

Received March 18, 2009, in final form June 11, 2009; Published online June 29, 2009

doi:10.3842/SIGMA.2009.066

\begin{abstract}
A study is made of 4-dimensional Lorentz manifolds which are projectively related, that is, whose Levi-Civita connections give rise to the same (unparameterised) geodesics. A brief review of some relevant recent work is provided and a list of new results connecting projective relatedness and the holonomy type of the Lorentz manifold in question is given. This necessitates a review of the possible holonomy groups for such manifolds which, in turn, requires a certain convenient classification of the associated curvature tensors. These reviews are provided.
\end{abstract}

Key words: projective structure; holonomy; Lorentz manifolds; geodesic equivalence

2000 Mathematics Subject Classification: 53C29; 53C22; 53C50

This paper is dedicated to the memory of Élie Cartan

\section{Introduction}

Élie Cartan (1869-1951) was one of the world's leading geometers and it is to his memory that the authors dedicate this paper. One of Cartan's main interests lay in the crucially important study of connections on manifolds and its applications to theoretical physics. The present paper will proceed in a similar vein by presenting a discussion of holonomy theory on a 4-dimensional manifold which admits a Lorentz metric. This leads naturally to applications in Einstein's general theory of relativity. Here the application will be to the projective structure of spacetimes and which itself is closely related to the principle of equivalence in Einstein's theory. A few weeks before this paper was begun, the authors learned, sadly, of the death of Élie Cartan's son, Henri, at the age of 104. Henri Cartan was also a world leader in geometry and this father and son combination laid down the foundations for a great deal of the research presently undertaken in differential geometry.

This paper will be arranged in the following way. Section 2 will be used to introduce general notation and in Section 3 a classification of the curvature tensor will be introduced and which will prove useful in what is to follow. Also included in Section 3 is a discussion of certain relationships between the metric, connection and curvature structures on a space-time. In Section 4 a review of holonomy theory will be given and, in particular, as it applies to 4-dimensional Lorentz manifolds. In Section 5, a discussion of projective structure will be presented. This will be followed, in Sections 6 and 7, with several theorems which show a tight relationship between projective relatedness and holonomy type. A brief summary of the paper is given in Section 8 .

\footnotetext{
*This paper is a contribution to the Special Issue "Élie Cartan and Differential Geometry". The full collection is available at http://www.emis.de/journals/SIGMA/Cartan.html
} 


\section{Notation and preliminary remarks}

Throughout this paper, $M$ will denote a 4-dimensional, Hausdorff, connected, smooth manifold which admits a smooth metric $g$ of Lorentz signature $(-,+,+,+)$. The pair $(M, g)$ is called a space-time. It follows that the (usual manifold) topology on $M$ is necessarily second countable [1] and hence paracompact. All structures on $M$ will be assumed smooth (where this is sensible). The unique symmetric Levi-Civita connection arising on $M$ through $g$ is denoted by $\nabla$ and, in a coordinate domain of $M$, its Christoffel symbols are written $\Gamma_{b c}^{a}$. The type $(1,3)$ curvature tensor associated with $\nabla$ is denoted by Riem and its (coordinate) components are written $R_{b c d}^{a}$. The Ricci tensor, Ricc, derived from Riem, has components $R_{a b}=R_{a c b}^{c}$ and $R=R_{a b} g^{a b}$ is the Ricci scalar. The Weyl type $(1,3)$ conformal tensor $C$ has components $C_{b c d}^{a}$ given by

$$
C_{b c d}^{a}=R_{b c d}^{a}-E_{b c d}^{a}-\frac{R}{12}\left(\delta_{c}^{a} g_{b d}-\delta_{d}^{a} g_{b c}\right),
$$

where $E$ is the tensor with components

$$
E_{b c d}^{a}=\frac{1}{12}\left(\tilde{R}_{c}^{a} g_{b d}-\tilde{R}_{d}^{a} g_{b c}+\delta_{c}^{a} \tilde{R}_{b d}-\delta_{d}^{a} \tilde{R}_{b c}\right)
$$

and where $\widetilde{\text { Ricc }}$ is the trace-free Ricci tensor with components $\widetilde{R}_{a b}=R_{a b}-\frac{R}{4} g_{a b}$. At any $m \in M$, the tensors $E$ and $\widetilde{\text { Ricc }}$ uniquely (algebraically) determine each other and $E=0 \Leftrightarrow \widetilde{\text { Ricc }}=0 \Leftrightarrow$ the Einstein space condition holding at $m$.

For $m \in M, T_{m} M$ denotes the tangent space to $M$ at $m$ and this will, for convenience, be identified with the cotangent space $T_{m}^{*} M$ to $M$ at $m$, through the metric $g(m)$ by index raising and lowering. Thus the liberty will be taken of using the same symbol for members of $T_{m} M$ and $T_{m}^{*} M$ which are so related and similarly for other tensor spaces. A tetrad (that is, a basis for $\left.T_{m} M\right) u, x, y, z \in T_{m} M$ is called orthonormal if and only if the only non-vanishing inner products between tetrad members are $-g(u, u)=g(x, x)=g(y, y)=g(z, z)=1$ and a tetrad $l, n, x, y \in T_{m} M$ is called null if and only if the only non-vanishing inner products between tetrad members are $g(l, n)=g(x, x)=g(y, y)=1$. In this case, $l$ and $n$ are null vectors. Another condition, the non-flat condition, will be imposed on $(M, g)$, meaning that Riem does not vanish over any non-empty open subset of $M$. This is a physical requirement and is there to prevent gravitational shielding in general relativity theory.

Let $\Lambda_{m} M$ denote the 6 -dimensional vector space of all tensor type $(2,0) 2$-forms at $m$. This vector space can be associated, using the metric $g(m)$, with the vector spaces of tensor type $(0,2) 2$-forms at $m$ and of type $(1,1)$ tensors at $m$ which are skew-self adjoint with respect to $g(m)$, through the component identifications

$$
\left(F \in \Lambda_{m} M\right) F^{a b}\left(=-F^{b a}\right) \rightarrow F_{a b} \equiv g_{a c} g_{b d} F^{c d} \rightarrow F_{b}^{a}=g_{c b} F^{a c} .
$$

Any member $F$ of any of these vector spaces will be referred to as a bivector (at $m$ ) and written symbolically as $F \in \Lambda_{m} M$. Any $F \in \Lambda_{m} M, F \neq 0$, has (matrix) rank 2 or 4 . If $F$ has rank 2 it is called simple and may be written in components as $F^{a b}=p^{a} q^{b}-q^{a} p^{b}$ for $p, q \in T_{m} M$. Although $F$ does not determine $p$ and $q$ it does uniquely determine the 2-dimensional subspace (referred to as a 2-space) of $T_{m} M$ spanned by $p$ and $q$ and which is called the blade of $F$. A simple bivector $F$ at $m$ is then called timelike, spacelike or null according as its blade is, respectively, a timelike, spacelike or null 2-space at $m$. If $F \in \Lambda_{m} M$ has rank 4 it is called non-simple and may be written as $F=G+H$ where $G$ and $H$ are simple bivectors with $G$ timelike and $H$ spacelike and where the blades of $G$ and $H$ are uniquely determined by $F$ and are orthogonal complements of each other. They will be collectively called the canonical pair of blades of $F$. The Hodge duality operator on bivectors is denoted by $*$. Then $F$ is simple if and 
only if $\stackrel{*}{F}$ is simple and the blades of $F$ and $\stackrel{*}{F}$ are orthogonal complements of each other. In this case $F$ is spacelike, respectively, timelike or null, if and only if $F^{*}$ is timelike, respectively, spacelike or null. If $F$ is simple and either spacelike or timelike, the blades of $F$ and $\stackrel{*}{F}$ are complementary (that is, their union spans $T_{m} M$ ). This is not the case if $F$ is null. In the above expression for a non-simple bivector $F, G$ and $H$ are (multiples of) duals of each other. For any bivector $F$ at $m, F$ and $\stackrel{*}{F}$ are independent bivectors at $m$ and $\stackrel{* *}{F}=-F$. If $F$ is a simple bivector at $m$ with $F^{a b}=p^{a} q^{b}-q^{a} p^{b}, p, q \in T_{m} M$, then $F$ (or its blade) is sometimes written as $p \wedge q$. It is convenient, on occasions, to use round and square brackets to denote the usual symmetrisation and skew-symmetrisation of indices, respectively. As a general remark on notation, both coordinate and coordinate-free notation will be used, depending on relative convenience. More details on these aspects of Minkowski geometry may be found, for example, in [2].

\section{Curvature structure of space-times}

Because of the algebraic symmetries of Riem, one may introduce the curvature maps $f$ and $\tilde{f}$ from the vector space of bivectors to itself at $m$ (recalling the liberties taken with this vector space in Section 2) by

$$
f: F^{a b} \rightarrow R_{b c d}^{a} F^{c d}, \quad \tilde{f}: F^{a b} \rightarrow R_{c d}^{a b} F^{c d} .
$$

The maps $f$ and $\tilde{f}$ are linear maps of equal rank, the latter being referred to as the curvature rank at $m$. Let $B_{m}$ denote the range space of $f$ or $\tilde{f}$ at $m$ (according to the agreed identification) so that $\operatorname{dim} B_{m}$ equals the curvature rank at $m$ and which, in turn, is $\leq 6$. This leads to a convenient algebraic classification of Riem at $m$ into five mutually exclusive and disjoint curvature classes (for further details, see [2]).

Class A This covers all possibilities not covered by classes B, C, D and $\mathbf{O}$ below. For this class, the curvature rank at $m$ is $2,3,4,5$ or 6 .

Class $\mathbf{B}$ This occurs when $\operatorname{dim} B_{m}=2$ and when $B_{m}$ is spanned by a timelike-spacelike pair of simple bivectors with orthogonal blades (chosen so that one is the dual of the other). In this case, one can choose a null tetrad $l, n, x, y \in T_{m} M$ such that these bivectors are $F=l \wedge n$ and $\stackrel{*}{F}=x \wedge y$ so that $F$ is timelike and $\stackrel{*}{F}$ is spacelike and then (using the algebraic identity $R_{a[b c d]}=0$ to remove cross terms) one has, at $m$,

$$
R_{a b c d}=\alpha F_{a b} F_{c d}+\beta \stackrel{*}{F}_{a b} \stackrel{*}{F}_{c d}
$$

for $\alpha, \beta \in \mathbb{R}, \alpha \neq 0 \neq \beta$.

Class $\mathbf{C}$ In this case $\operatorname{dim} B_{m}=2$ or 3 and $B_{m}$ may be spanned by independent simple bivectors $F$ and $G$ (or $F, G$ and $H$ ) with the property that there exists $0 \neq r \in T_{m} M$ such that $r$ lies in the blades of $\stackrel{*}{F}$ and $\stackrel{*}{G}$ ( or $\stackrel{*}{F}, \stackrel{*}{G}$ and $\stackrel{*}{H}$ ). Thus $F_{a b} r^{b}=G_{a b} r^{b}\left(=H_{a b} r^{b}\right)=0$ and $r$ is then unique up to a multiplicative non-zero real number.

Class $\mathbf{D}$ In this case $\operatorname{dim} B_{m}=1$. If $B_{m}$ is spanned by the bivector $F$ then, at $m$,

$$
R_{a b c d}=\alpha F_{a b} F_{c d}
$$

for $0 \neq \alpha \in \mathbb{R}$ and $R_{a[b c d]}=0$ implies that $F_{a[b} F_{c d]}=0$ from which it may be checked that $F$ is necessarily simple.

Class $\mathbf{O}$ In this case Riem vanishes at $m$. 
It is remarked that this classification is pointwise and may vary over $M$. The subset of $M$ consisting of points at which the curvature class is $\mathbf{A}$ is an open subset of $M$ [2, p. 393] and the analogous subset arising from the class $\mathbf{O}$ is closed (and has empty interior in the manifold topology of $M$ if $(M, g)$ is non-flat). It is also useful to note that the equation $R_{a b c d} k^{d}=0$ at $m$ has no non-trivial solutions if the curvature class at $m$ is $\mathbf{A}$ or $\mathbf{B}$, a unique independent solution (the vector $r$ above) if the curvature class at $m$ is $\mathbf{C}$ and two independent solutions if the curvature class at $m$ is $\mathbf{D}$ (and which span the blade of $\stackrel{*}{F}$ in $(3.3)$ ). If $\operatorname{dim} B_{m} \geq 4$ the curvature class at $m$ is $\mathbf{A}$. If $(M, g)$ has the same curvature class at each $m \in M$ it will be referred to as being of that class.

Related to this classification scheme is the following result which will prove useful in what is to follow. The details and proof can be found in $[3,4,5,2]$.

Theorem 3.1. Let $(M, g)$ be a space-time, let $m \in M$ and let $h$ be a second order, symmetric, type $(0,2)$ (not necessarily non-degenerate) tensor at $m$ satisfying $h_{a e} R_{b c d}^{e}+h_{b e} R_{a c d}^{e}=0$. Then, with all tensor index movements and all orthogonality statements made using the metric $g$;

(i) if the curvature class of $(M, g)$ at $m \in M$ is $\mathbf{D}$ and $u, v \in T_{m} M$ span the 2-space at $m$ orthogonal to $F$ in (3.3) (that is $u \wedge v$ is the blade of $\stackrel{*}{F}$ ) there exists $\phi, \mu, \nu, \lambda \in \mathbb{R}$ such that, at $m$,

$$
h_{a b}=\phi g_{a b}+\mu u_{a} u_{b}+\nu v_{a} v_{b}+\lambda\left(u_{a} v_{b}+v_{a} u_{b}\right) ;
$$

(ii) if the curvature class of $(M, g)$ at $m \in M$ is $\mathbf{C}$ there exists $r \in T_{m} M$ (the vector appearing in the above definition of class $\mathbf{C})$ and $\phi, \lambda \in \mathbb{R}$ such that, at $m$,

$$
h_{a b}=\phi g_{a b}+\lambda r_{a} r_{b}
$$

(iii) if the curvature class of $(M, g)$ at $m \in M$ is $\mathbf{B}$ there exists a null tetrad $l, n, x, y$ (that appearing in the above definition of class $\mathbf{B})$ and $\phi, \lambda \in \mathbb{R}$ such that, at $m$,

$$
h_{a b}=\phi g_{a b}+\lambda\left(l_{a} n_{b}+n_{a} l_{b}\right)=(\phi+\lambda) g_{a b}-\lambda\left(x_{a} x_{b}+y_{a} y_{b}\right)
$$

(iv) if the curvature class of $(M, g)$ at $m \in M$ is $\mathbf{A}$ there exists $\phi \in \mathbb{R}$ such that, at $m$,

$$
h_{a b}=\phi g_{a b}
$$

The proof is essentially based on the obvious fact that the range $B_{m}$ of the map $f$ in (3.1) which, by the algebraic symmetries of the curvature Riem of $(M, g)$, consists entirely of members which are skew-self adjoint with respect to $g$, must likewise consist entirely of members skew-self adjoint with respect to $h$. Thus each $F \in B_{m}$ satisfies

$$
g_{a c} F_{b}^{c}+g_{b c} F_{a}^{c}=0, \quad h_{a c} F_{b}^{c}+h_{b c} F_{a}^{c}=0 .
$$

It is a consequence of (3.8) that the blade of $F$ (if $F$ is simple) and each of the canonical pair of blades of $F$ (if $F$ is non-simple) are eigenspaces of $h$ with respect to $g$, that is, for $F$ simple, any $k$ in the blade of $F$ satisfies $h_{a b} k^{b}=\omega g_{a b} k^{b}$ where the eigenvalue $\omega \in \mathbb{R}$ is independent of $k$, and similarly for each of the canonical blades if $F$ is non-simple (but with possibly different eigenvalues for these blades) [2, 3, 4].

It is remarked that if $g^{\prime}$ is another metric on $M$ whose curvature tensor Riem' equals the curvature tensor Riem of $g$ everywhere on $M$ then the conditions of this theorem are satisfied for $h=g^{\prime}(m)$ at each $m \in M$ and so the conclusions also hold except that now one must 
add the restriction $\phi \neq 0$ in each case to preserve the non-degeneracy of $g^{\prime}$ at $m$ and maybe some restrictions on $\phi, \mu, \nu$ and $\lambda$ if the signature of $g^{\prime}$ is prescribed. If $(M, g)$ is of class $\mathbf{A}$, (3.7) gives $g^{\prime}=\phi g$ and the Bianchi identity may be used to show that $\phi$ is constant on $M[4,2]$. When Theorem 3.1 is applied to another metric $g^{\prime}$ on $M$ in this way, it consolidates the curvature classification scheme which preceded it. To see this note that $g^{\prime}$ need not have Lorentz signature $(-,+,+,+)$ but, if this is insisted upon, the curvature classification scheme, including the nature (timelike, spacelike or null) of $F$ and $\stackrel{*}{F}$ in class $\mathbf{B}, r$ in class $\mathbf{C}$ and $F$ in class $\mathbf{D}$ is the same whether taken for (the common curvature tensor) Riem with $g$ or Riem with $g^{\prime}$. In this sense it is a classification of Riem, independent of the metric generating Riem [6].

\section{Holonomy theory}

Let $(M, g)$ be a space-time with Levi-Civita connection $\nabla$ and let $m \in M$. The connection $\nabla$ is a complicated object but one particularly pleasing feature of it lies in the following construction. Let $m \in M$ and for $1 \leq k \leq \infty$ let $C_{k}(m)$ denote the set of all piecewise $C^{k}$ closed curves starting and ending at $m$. If $c \in C_{k}(m)$ let $\tau_{c}$ denote the vector space isomorphism of $T_{m} M$ obtained by parallel transporting, using $\nabla$, each member of $T_{m} M$ along $c$. Using a standard notation associated with curves one defines, for curves $c, c_{0}, c_{1}, c_{2} \in C_{k}(m)$, with $c_{0}$ denoting a constant curve at $m$, the identity map $\tau_{c_{0}}$ on $T_{m} M$, the inverse $\tau_{c}^{-1} \equiv \tau_{c^{-1}}$ and product $\tau_{c_{1}} \cdot \tau_{c_{2}} \equiv \tau_{c_{1} \cdot c_{2}}$ to put a group structure on $\left\{\tau_{c}: c \in C_{k}(m)\right\}$, making it a subgroup of $G \equiv G L\left(T_{m} M\right)(=G L(4, \mathbb{R}))$, called the $k$-holonomy group of $M$ at $m$ and denoted by $\Phi_{k}(m)$. In fact, since $M$ is connected and also a manifold, it is also path connected and, as a consequence, it is easily checked that, up to an isomorphism, $\Phi_{k}(m)$ is independent of $m$. Less obvious is the fact that $\Phi_{k}(m)$ is independent of $k(1 \leq k \leq \infty)$ and thus one arrives at the holonomy group $\Phi$ (of $\nabla$ ) on $M$. Further details may be found in [7] and a summary in [2].

One could repeat the above operations, but now only using curves homotopic to zero. the above independence of $m$ and $k$ still holds and one arrives at the restricted holonomy group $\Phi^{0}$ of $M$. It can now be proved that $\Phi$ and $\Phi^{0}$ are Lie subgroups of $G$, with $\Phi^{0}$ connected, and that $\Phi^{0}$ is the identity component of $\Phi$ [7]. Clearly, if $M$ is simply connected, $\Phi=\Phi^{0}$ and then $\Phi$ is a connected Lie subgroup of $G$. The common Lie algebra $\phi$ of $\Phi$ and $\Phi^{0}$ is called the holonomy algebra. The connection $\nabla$ can then be shown to be flat (that is, Riem vanishes on $M$ ) if and only if $\phi$ is trivial and this, in turn, is equivalent to $\Phi^{0}$ being trivial.

For a space-time $(M, g)$, however, one has the additional information that $\nabla$ is compatible with the metric $g$, that is, $\nabla g=0$. Thus each map $\tau_{c}$ on $T_{m} M$ preserves inner products with respect to $g(m)$. It follows that $\Phi$ is (isomorphic to) a subgroup of the Lorentz group $\mathcal{L}$, where $\mathcal{L}=\left\{A \in G L(4, \mathbb{R}): A \eta A^{T}=\eta\right\}$ where $A^{T}$ denotes the transpose of $A$ and $\eta$ is the Minkowski metric, $\eta=\operatorname{diag}(-1,1,1,1)$. Now $\Phi$ is a Lie subgroup of $G L(4, \mathbb{R})$ and $\mathcal{L}$ can be shown to be a 6-dimensional Lie subgroup of $G L(4, \mathbb{R})$ which is a regular submanifold of $G L(4, \mathbb{R})$ (that is, the (sub)manifold topology on $\mathcal{L}$ equals its induced topology from $G L(4, \mathbb{R})$ ). It follows that $\Phi$ is a Lie subgroup of $\mathcal{L}$ (see e.g. [2]) and hence that $\Phi^{0}$ (or $\Phi$, if $M$ is simply connected) is a connected Lie subgroup of the identity component, $\mathcal{L}_{0}$, of $\mathcal{L}$. Thus the holonomy algebra $\phi$ can be identified with a subalgebra of the Lie algebra $L$ of $\mathcal{L}$, the Lorentz algebra. The oneto-one correspondence between the subalgebras of $L$ and the connected Lie subgroups of $\mathcal{L}_{0}$ shows that the Lie group $\Phi^{0}$ (or $\Phi$, if $M$ is simply connected) is determined by the subalgebra of $L$ associated with $\phi$. Fortunately, the subalgebra structure of $L$ is well-known and can be conveniently represented as follows. Let $m \in M$ and choose a basis for $T_{m} M$ (together with its dual basis) for which $g(m)$ has components equal to, say, $g_{a b}$. Then $\mathcal{L}$ is isomorphic to $\left\{A \in G L(4, \mathbb{R}): A g(m) A^{T}=g(m)\right\}$ and $L$ can then be represented as the subset of $M_{4} \mathbb{R}$ each member of which, if regarded as the set of components of a type $(1,1)$ tensor at $m$ in this 
Table 1. Holonomy algebras. For types $R_{5}$ and $R_{12} 0 \neq \omega \in \mathbb{R}$. Every potential holonomy algebra except $R_{5}$ (for which the curvature tensor fails to satisfy the algebraic Bianchi identity) can occur as an actual holonomy algebra, see, e.g. [2]). There is also a type $R_{1}$, when $\phi$ is trivial and $(M, g)$ is flat, but this trivial type is omitted. Type $R_{15}$ is the "general" type, when $\phi=L$.

\begin{tabular}{|c|c|c|c|c|c|}
\hline Type & Dimension & Basis & $\begin{array}{c}\text { Curvature } \\
\text { Class }\end{array}$ & $\begin{array}{c}\text { Recurrent } \\
\text { vector fields }\end{array}$ & $\begin{array}{c}\text { Constant } \\
\text { vector fields }\end{array}$ \\
\hline$R_{2}$ & 1 & $l \wedge n$ & $\mathbf{D}$ or $\mathbf{O}$ & $\{l\},\{n\}$ & $\langle x, y\rangle$ \\
$R_{3}$ & 1 & $l \wedge x$ & $\mathbf{D}$ or $\mathbf{O}$ & - & $\langle l, y\rangle$ \\
$R_{4}$ & 1 & $x \wedge y$ & $\mathbf{D}$ or $\mathbf{O}$ & - & $\langle l, n\rangle$ \\
$R_{5}$ & 1 & $l \wedge n+\omega x \wedge y$ & - & - & - \\
$R_{6}$ & 2 & $l \wedge n, l \wedge x$ & $\mathbf{C}, \mathbf{D}$ or $\mathbf{O}$ & $\{l\}$ & $\langle y\rangle$ \\
$R_{7}$ & 2 & $l \wedge n, x \wedge y$ & $\mathbf{B}, \mathbf{D}$ or $\mathbf{O}$ & $\{l\},\{n\}$ & - \\
$R_{8}$ & 2 & $l \wedge x, l \wedge y$ & $\mathbf{C}, \mathbf{D}$ or $\mathbf{O}$ & - & $\langle l\rangle$ \\
$R_{9}$ & 3 & $l \wedge n, l \wedge x, l \wedge y$ & $\mathbf{A}, \mathbf{C}, \mathbf{D}$ or $\mathbf{O}$ & $\{l\}$ & - \\
$R_{10}$ & 3 & $l \wedge n, l \wedge x, n \wedge x$ & $\mathbf{C}, \mathbf{D}$ or $\mathbf{O}$ & - & $\langle y\rangle$ \\
$R_{11}$ & 3 & $l \wedge x, l \wedge y, x \wedge y$ & $\mathbf{C}, \mathbf{D}$ or $\mathbf{O}$ & - & $\langle l\rangle$ \\
$R_{12}$ & 3 & $l \wedge x, l \wedge y, l \wedge n+\omega(x \wedge y)$ & $\mathbf{A}, \mathbf{C}, \mathbf{D}$ or $\mathbf{O}$ & $\{l\}$ & - \\
$R_{13}$ & 3 & $x \wedge y, y \wedge z, x \wedge z$ & $\mathbf{C}, \mathbf{D}$ or $\mathbf{O}$ & - & $\langle u\rangle$ \\
$R_{14}$ & 4 & $l \wedge n, l \wedge x, l \wedge y, x \wedge y$ & any & $\{l\}$ & - \\
$R_{15}$ & 6 & $L$ & any & - & - \\
\hline
\end{tabular}

basis, is skew-self adjoint with respect to the matrix $g_{a b}(m)$ (that is, its components $F_{b}^{a}$ satisfy an equation like the first in $(3.8)$ with respect to $g(m)$ ). Thus one can informally identify $L$ with this collection of bivectors, usually, written in type $(2,0)$ form. The binary operation on $L$ is that induced from the Lie algebra $M_{4} \mathbb{R}$ of $G L(4, \mathbb{R})$ and is matrix commutation. Such a representation of $L$ is well-known and has been classified into fifteen convenient types [8] (for details of the possible holonomy types most relevant for the physics of general relativity see [9]). It is given in the first three columns of Table 1 using either a null tetrad $l, n, x, y$ or an orthonormal tetrad $u, x, y, z$ to describe a basis for each subalgebra.

Now suppose $M$ is simply connected. This condition is not always required but is imposed in this section for convenience. It can always be assumed in local work, for example, in some connected, simply connected coordinate domain. In this case $\Phi=\Phi^{0}$ and is connected and $\Phi$ will be referred to according to its Lie algebra label as in Table 1. Two important results can now be mentioned in connection with the first column of Table 1. First, it turns out $[10,11,2]$ that if $m \in M$ there exists $0 \neq k \in T_{m} M$ such that $F_{b}^{a} k^{b}=0$ for each $F \in \phi$ if and only if $M$ admits a global, covariantly constant, smooth vector field whose value at $m$ is $k$. A basis for the vector space of such vector fields on $M$ for each holonomy type is given inside \langle\rangle brackets in the final column of Table 1. Second, there exists $0 \neq k \in T_{m} M$ such that $k$ is an eigenvector of each $F \in \phi$ but with at least one associated eigenvalue not zero if and only if $M$ admits a global smooth properly recurrent vector field $X$ whose value at $m$ is $k$, that is, a global nowhere zero vector fiel $X$ on $M$ satisfying $\nabla X=X \otimes w$ for some global, smooth covector field $w$ on $M$ (the recurrence 1-form) and such that no function $\alpha: M \rightarrow \mathbb{R}$ exists such that $\alpha$ is nowhere zero on $M$ and $\alpha X$ is covariantly constant on $M$. It follows from the existence of one nonzero eigenvalue at $m$ in the above definition that $X$ is necessarily null (by the skew-self adjoint property of the members of $\phi$ ). The independent properly recurrent vector fields are listed for each holonomy type in \{\} brackets in the second from last column of Table 1. (It is remarked here that a nowhere zero vector field $Y$ on $M$ is called recurrent if $\nabla Y=Y \otimes r$ for some global covector field $r$ on $M$. In this case $r$ could be identically zero and so (non-trivial) covariantly constant vector fields are, in this sense, recurrent. In fact, any non-null recurrent vector field or 
any recurrent vector field on a manifold with positive definite metric can be globally scaled to be nowhere zero and covariantly constant because if $Y$ is any such vector field and $\nabla Y=Y \otimes r$, $\alpha Y$ is covariantly constant, where $\alpha=\exp \left(-\frac{1}{2} \log (g(Y, Y))\right)$.)

A recurrent vector field is easily seen to define a 1-dimensional distribution on $M$ which is preserved by parallel transport. There is an important generalisation of this concept. Let $m \in M$ and $V$ a non-trivial proper subspace of $T_{m} M$. Suppose $\tau_{c}(V)=V$ for each $\tau_{c}$ arising from $c \in C_{k}(m)$ at $m$. Then $V$ is holonomy invariant and gives rise in an obvious way to a smooth distribution on $M$ which is, in fact, integrable [7]. Clearly, if $V \subset T_{m} M$ is holonomy invariant then so is the orthogonal complement, $V^{\perp}$, of $V$. If such a $V$ exists the holonomy group $\Phi$ of $M$ is called reducible (otherwise, irreducible). (This concept of holonomy reducibility is a little more complicated in the case of a Lorentz metric than in the positive definite case due to the possibility of null holonomy invariant subspaces giving a weaker form of reducibility. This will not be pursued any further here, more details being available in [12] and summaries in $[2,9,11]$.) Thus, for example, in the notation of Table 1 the holonomy type $R_{2}$ admits two 1-dimensional null holonomy invariant subspaces spanned by $l$ and $n$ and which give rise to two null properly recurrent vector fields and infinitely many 1-dimensional spacelike holonomy invariant subspaces spanned by the infinitely many covariantly constant vector fields in $\langle x, y\rangle$. For the holonomy type $R_{7}$, two 1-dimensional null holonomy invariant subspaces exist and which give rise to two independent properly recurrent null vector fields as in the previous case, together with a 2-dimensional spacelike one orthogonal to each of the null ones. For holonomy types $R_{10}$, $R_{11}$ and $R_{13}$ one has a 1-dimensional holonomy invariant subspace, spanned by a covariantly constant vector field in each case, together with its orthogonal complement. These holonomy decompositions will be useful in Sections 6 and 7 . In the event that $M$ is not simply connected, the vector fields determined by the holonomy and described above may not exist globally but do exist locally over some open, connected and simply connected neighbourhood of any point.

It is remarked here that if $(M, g)$ is of curvature class $\mathbf{B}$ it can be shown that it must in fact be of holonomy type $R_{7}[13,2]$.

It is useful, at this point, to introduce the infinitesimal holonomy group $\Phi_{m}^{\prime}$, of $(M, g)$ at each $m \in M$. Using a semi-colon to denote a $\nabla$-covariant derivative, consider, in some coordinate neighbourhood of $m$, the following matrices for $(M, g)$ at $m$

$$
R_{b c d}^{a} X^{c} Y^{d}, \quad R_{b c d ; e}^{a} X^{c} Y^{d} Z^{e}, \quad \ldots
$$

for $X, Y, Z, \ldots \in T_{m} M$. It turns out that the collection (4.1) spans a subalgebra of the holonomy algebra $\phi$ (and hence only a finite number of terms arise in (4.1) [7]). This algebra is called the infinitesimal holonomy algebra at $m$ and is denoted by $\phi_{m}^{\prime}$. The unique connected Lie subgroup of $\Phi$ that it gives rise to is the infinitesimal holonomy group $\Phi_{m}^{\prime}$ at $m$. This is useful in that it says that the range space of the map $f$ in (3.1) is, at each $m \in M$, (isomorphic as a vector space to) a subspace of $\phi$. This gives a restriction, when $\phi$ is known, on the expression for Riem at each $m$ and hence on its curvature class at $m$. This restriction is listed in the fourth column of Table 1. Thus if the holonomy type of $(M, g)$ is $R_{2}, R_{3}$ or $R_{4}$ its curvature class is $\mathbf{O}$ or $\mathbf{D}$ at each $m \in M$ whilst if it is $R_{6}, R_{8}, R_{10}, R_{11}$ or $R_{13}$ it is $\mathbf{O}, \mathbf{D}$ or $\mathbf{C}$, if $R_{7}$ it is $\mathbf{O}, \mathbf{D}$ or $\mathbf{B}$, if $R_{9}$ or $R_{12}$ it is $\mathbf{O}, \mathbf{D}, \mathbf{C}$ or $\mathbf{A}$ and if $R_{14}$ or $R_{15}$ it could be any curvature class. A useful relationship between the various algebras $\phi_{m}^{\prime}$, the algebra $\phi$ and the curvature class (through the range space $B_{m}$ ) at each $m \in M$ is provided by the Ambrose-Singer theorem [14] (see also [7]).

\section{$5 \quad$ Projective structure}

One aspect of differential geometry that has been found interesting both for pure geometers and physicists working in general relativity theory is that of projective structure. For general 
relativity it is clearly motivated by the Newton-Einstein principle of equivalence. In this section it will take the form described in the following question; for a space-time $(M, g)$ with LeviCivita connection $\nabla$, if one knows the paths of all the unparameterised geodesics (that is, only the geodesic paths in $M)$ how tightly is $\nabla$ determined? Put another way, let $(M, g)$ and $\left(M, g^{\prime}\right)$ be space-times with respective Levi-Civita connections $\nabla$ and $\nabla^{\prime}$, such that the sets of geodesic paths of $\nabla$ and $\nabla^{\prime}$ coincide (and let it be agreed that $\nabla$ and $\nabla^{\prime}$ (or $g$ and $g^{\prime}$ ) are then said to be projectively related (on $M)$ ). What can be deduced about the relationship between $\nabla$ and $\nabla^{\prime}$ (and between $g$ and $g^{\prime}$ )? (And it is, perhaps, not surprising that some reasonable link should exist between projective relatedness and holonomy theory.) In general, $\nabla$ and $\nabla^{\prime}$ may be expected to differ but it turns out that in many interesting situations they are necessarily equal. If $\nabla=\nabla^{\prime}$ is the result, holonomy theory can also describe precisely, the (simple) relationship between $g$ and $g^{\prime}[15,2]$.

If $\nabla$ and $\nabla^{\prime}$ are projectively related then there exists a uniquely defined global smooth 1-form field $\psi$ on $M$ such that, in any coordinate domain of $M$, the respective Christoffel symbols of $\nabla$ and $\nabla^{\prime}$ satisfy $[16,17]$

$$
\Gamma_{b c}^{\prime a}-\Gamma_{b c}^{a}=\delta_{b}^{a} \psi_{c}+\delta_{c}^{a} \psi_{b}
$$

It is a consequence of the fact that $\nabla$ and $\nabla^{\prime}$ are metric connections that $\psi$ is a global gradient on $M$ [16]. Equation (5.1) can, by using the identity $\nabla^{\prime} g^{\prime}=0$, be written in the equivalent form

$$
g_{a b ; c}^{\prime}=2 g_{a b}^{\prime} \psi_{c}+g_{a c}^{\prime} \psi_{b}+g_{b c}^{\prime} \psi_{a}
$$

(recalling that a semi-colon denotes covariant differentiation with respect to $\nabla$ ). Equation (5.1) reveals a simple relation between the type $(1,3)$ curvature tensors Riem and Riem ${ }^{\prime}$ of $\nabla$ and $\nabla^{\prime}$, respectively, given by

$$
R_{b c d}^{\prime a}=R_{b c d}^{a}+\delta_{d}^{a} \psi_{b c}-\delta_{c}^{a} \psi_{b d} \quad\left(\Rightarrow R_{a b}^{\prime}=R_{a b}-3 \psi_{a b}\right),
$$

where $\psi_{a b} \equiv \psi_{a ; b}-\psi_{a} \psi_{b}=\psi_{b a}$ and where $R_{a b}^{\prime} \equiv R_{a c b}^{\prime c}$ are the Ricci tensor components of $\nabla^{\prime}$. It can now be shown that if $\nabla$ and $\nabla^{\prime}$ are projectively related, the following type $(1,3)$ Weyl projective tensor, $W$, is the same for each of them [18]

$$
W_{b c d}^{a}=R_{b c d}^{a}+\frac{1}{3}\left(\delta_{d}^{a} R_{b c}-\delta_{c}^{a} R_{b d}\right) .
$$

A particularly important case of such a study arises where the original pair $(M, g)$ is a spacetime which is also an Einstein space so that the tensor $E$ in (2.2) is identically zero on $M$. Such a situation has been discussed in several places [19, 20, 21, 22, 23, 24] in connection with the principle of equivalence. The particular case which is, perhaps, of most importance in general relativity arises when the Ricci scalar vanishes and then $(M, g)$ is a vacuum (Ricci flat) space-time and this is discussed in [22, 24]. It turns out that if $(M, g)$ is a space-time which is a (general) Einstein space (and with the non-flat assumption temporarily dropped) and if $g^{\prime}$ is another metric on $M$ projectively related to $g$ then either $(M, g)$ and $\left(M, g^{\prime}\right)$ are each of constant curvature, or the Levi-Civita connections $\nabla$ and $\nabla^{\prime}$ of $g$ and $g^{\prime}$, respectively, are equal. In the event that neither space-time is of constant curvature, (and so $\nabla^{\prime}=\nabla$ ) an argument from holonomy theory can be used to show that, generically, $\left(M, g^{\prime}\right)$ is also an Einstein space and that $g^{\prime}=c g(0 \neq c \in \mathbb{R})$. Although examples exist where each of these conclusions fail, $g^{\prime}$ always has Lorentz signature (up to an overall minus sign). If, in addition, $(M, g)$ is assumed vacuum and the non-flat condition is imposed, then, necessarily, $\nabla=\nabla^{\prime}$ and, with one very special case excluded, $g^{\prime}=c g$ on $M(0 \neq c \in \mathbb{R})$ (and so $\left(M, g^{\prime}\right)$ is also vacuum). For this case $g^{\prime}$ has the same signature as $g$ (up to an overall minus sign $[22,24]$ ). This result is relevant for general relativity theory. A similar restrictive result for space-times of certain holonomy types will be established in the next two sections. 
The formalism described above characterises the relationship between the connections and metrics of two projectively related space-times $(M, g)$ and $\left(M, g^{\prime}\right)$. However, it is convenient to rewrite them in a different way using the Sinyukov transformation [20]. Thus, with this projective relatedness assumed, one takes advantage of the fact that the 1-form $\psi$ in (5.1) is necessarily a global gradient by writing $\psi=d \chi$ for some smooth function $\chi: M \rightarrow \mathbb{R}$. Then the pair $g^{\prime}$ and $\psi$ above are replaced by a type $(0,2)$ symmetric tensor field $a$ and a 1 -form field $\lambda$ on $M$ which are given in terms of $g^{\prime}$ and $\psi$ by

$$
a_{a b}=e^{2 \chi} g^{\prime c d} g_{a c} g_{b d}, \quad \lambda_{a}=-e^{2 \chi} \psi_{b} g^{\prime b c} g_{a c} \quad\left(\Rightarrow \lambda_{a}=-a_{a b} \psi^{b}\right),
$$

where an abuse of notation has been used in that $g^{\prime a b}$ denotes the contravariant components of $g^{\prime}$ (and not the tensor $g_{a b}^{\prime}$ with indices raised using $g$ ) so that $g_{a c}^{\prime} g^{\prime c b}=\delta_{a}^{b}$. Then (5.5) may be inverted to give

$$
g^{\prime a b}=e^{-2 \chi} a_{c d} g^{a c} g^{b d}, \quad \psi_{a}=-e^{-2 \chi} \lambda_{b} g^{b c} g_{a c}^{\prime} .
$$

The idea is that if $g$ and $g^{\prime}$ are projectively related metrics on $M$, so that (5.2) holds for some 1 -form $\psi(=d \chi)$, then $a$ and $\lambda$ as defined in (5.5) can be shown, after a short calculation, to satisfy Sinjukov's equation

$$
a_{a b ; c}=g_{a c} \lambda_{b}+g_{b c} \lambda_{a} .
$$

From (5.5) it follows that $a$ is non-degenerate and from (5.7), after a contraction with $g^{a b}$, that $\lambda$ is a global gradient on $M$ (in fact, of $\frac{1}{2} a_{a b} g^{a b}$ ).

In practice, when asking which pairs $\left(g^{\prime}, \nabla^{\prime}\right)$ are projectively related to some original pair $(g, \nabla)$ on $M$, it is often easier to use (5.7) to attempt to find $a$ and $\lambda$ rather than (5.2) to find $g^{\prime}$ and $\psi$. But then one must be able to convert back from $a$ and $\lambda$ to $g^{\prime}$ and $\psi$. To do this one first assumes that such a non-degenerate tensor $a$ and a 1-form $\lambda$ are given on $M$ and which together satisfy (5.7) and so one may define a symmetric non-degenerate type $(2,0)$ tensor $a^{-1}$ on $M$ which, at each $m \in M$, is the inverse of $a\left(a_{a c}\left(a^{-1}\right)^{c b}=\delta_{a}^{b}\right)$. Then raising and lowering indices on $a$ and $a^{-1}$ with $g$ in the usual way, so that $a_{a c}^{-1} a^{c b}=\delta_{a}^{b}$, one defines a global 1-form $\psi$ on $M$ by $\psi_{a}=-a_{a b}^{-1} \lambda^{b}$ (and so $\lambda_{a}=-a_{a b} \psi^{b}$ ). It follows that $\psi$ is a global gradient on $M$. To see this first differentiate the condition $a_{a c}^{-1} a^{c b}=\delta_{a}{ }^{b}$ and use (5.7) to find, after a short calculation,

$$
a_{a b ; c}^{-1}=a_{a c}^{-1} \psi_{b}+a_{b c}^{-1} \psi_{a} .
$$

Now define a smooth, symmetric connection $\nabla^{\prime \prime}$ on $M$ by decreeing that, in any coordinate domain, its Christoffel symbols are given by $\Gamma^{\prime \prime}$ where

$$
\Gamma_{b c}^{\prime \prime a}=\Gamma_{b c}^{a}-\psi^{a} g_{b c}
$$

Then it is easily checked from (5.8), (5.9) and the equation $\lambda_{a}=-a_{a b} \psi^{b}$ that $\nabla^{\prime \prime} a=0$ and so if the everywhere non-degenerate tensor $a$ is regarded as a metric on $M, \nabla^{\prime \prime}$ is its Levi-Civita connection. But a contraction of (5.9) over the indices $a$ and $c$ gives, from a standard formula for Christoffel symbols

$$
\Gamma_{b a}^{\prime \prime a}-\Gamma_{b a}^{a}=\frac{\partial}{\partial x^{b}}\left(\frac{1}{2} \ln \left(\frac{|\operatorname{det} a|}{|\operatorname{det} g|}\right)\right)=-\psi_{b}
$$

and so $\psi$ is seen to be a global gradient on $M$. (The authors have recently discovered that Sinyukov [20] had established the same result by a method involving the direct construction of the connection of the tensor $a^{-1}$.) Writing $\chi$ for this potential function, so that $\psi=d \chi$, one defines a metric $g^{\prime}$ on $M$ by $g^{\prime}=e^{2 \chi} a$. Then (5.8) can be used to show that $g^{\prime}$ and $\psi$ 
satisfy (5.2) and hence that $g^{\prime}$ is projectively related to $g$. It is easily checked that the tensors $\psi$ and $g^{\prime}$ thus found satisfy (5.5) and (5.6). [It is remarked that if $\psi$ is replaced by $\lambda$ and the metric $g$ by the tensor $a^{-1}$ in (5.9) the connection $\nabla^{\prime \prime \prime}$ thus defined satisfies $\nabla^{\prime \prime \prime} a^{-1}=0$. In fact, $\nabla=\nabla^{\prime \prime} \Leftrightarrow \nabla=\nabla^{\prime \prime \prime} \Leftrightarrow \nabla=\nabla^{\prime}$.] Since any solution pair $\left(g^{\prime}, \psi\right)$ of (5.2) leads to a pair $(a, \lambda)$ satisfying (5.7) it follows that all projectively related metrics $g^{\prime}$ together with their attendant 1 -forms $\psi$ will be found if all pairs $(a, \lambda)$ can be found and which, together, satisfy (5.7).

For the finding of the general solution of (5.7) a useful result arises by applying the Ricci identity to $a$ and using (5.7) to get

$$
\left(a_{a b ; c d}-a_{a b: d c}=\right) a_{a e} R_{b c d}^{e}+a_{b e} R_{a c d}^{e}=g_{a c} \lambda_{b d}+g_{b c} \lambda_{a d}-g_{a d} \lambda_{b c}-g_{b d} \lambda_{a c}
$$

where $\lambda_{a b}=\lambda_{a ; b}=\lambda_{b a}$. This leads to the following lemma (which is a special case of a more detailed result in [22]) and for which a definition is required. Suppose $m \in M$, that $F \in \Lambda_{m} M$ and that the curvature tensor Riem of $(M, g)$ satisfies $R^{a b}{ }_{c d} F^{c d}=\alpha F^{a b}(\alpha \in \mathbb{R})$ at $m$ so that $F$ is a (real) eigenvector of the map $\tilde{f}$ in (3.1). Then $F$ is called a (real) eigenbivector of Riem at $m$ with eigenvalue $\alpha$.

Lemma 5.1. Let $(M, g)$ and $\left(M, g^{\prime}\right)$ be space-times with $g$ and $g^{\prime}$ projectively related. Suppose at $m \in M$ that $F \in \Lambda_{m} M$ is a (real) eigenbivector of Riem of $(M, g)$ with zero eigenvalue (so that $F$ is in the kernel, $\operatorname{ker} \tilde{f}$, of $\tilde{f}$ in (3.1)). Then the blade of $F$ (if $F$ is simple) or each of the canonical pair of blades of $F$ (if $F$ is non-simple) is an eigenspace of the symmetric tensor $\lambda_{a b}$ with respect to $g$ at $m$. (That is, if $p \wedge q$ is in any of these blades $\left(p, q \in T_{m} M\right)$ there exists $\mu \in \mathbb{R}$ such that for any $\left.k \in p \wedge q, \lambda_{a b} k^{b}=\mu g_{a b} k^{b}\right)$. In particular,

(i) suppose that, at $m$, the collection of all the blades of all simple members of ker $\tilde{f}$ and all the canonical blade pairs of all the non-simple members of ker $\tilde{f}$ (and which are each eigenspaces of $\left.\lambda_{a ; b}\right)$ are such that they force $T_{m} M$ to be an eigenspace of $\lambda_{a ; b}$. Then $\lambda_{a ; b}$ is proportional to $g_{a b}$ at $m$.

(ii) If condition ( $i)$ is satisfied at each $m^{\prime}$ in some connected open neighbourhood $U$ of $m$, then, on $U$ and for some $c \in \mathbb{R}$,
(a) $\lambda_{a b}=c g_{a b}$
(b) $\lambda_{d} R_{a b c}^{d}=0$,
(c) $a_{a e} R_{b c d}^{e}+a_{b e} R_{a c d}^{e}=0$.

Proof. First contract (5.11) with $F^{c d}$ and use $R_{b c d}^{a} F^{c d}=0$ to get

$$
g_{a e} F^{c e} \lambda_{b c}+g_{b e} F^{c e} \lambda_{a c}-g_{a e} F^{e d} \lambda_{b d}-g_{b e} F^{e d} \lambda_{a d}=0,
$$

which rearranges, after cancellation of a factor 2 , as

$$
\lambda_{a c} F_{b}^{c}+\lambda_{b c} F_{a}^{c}=0
$$

where $F_{b}^{a}=F^{a c} g_{c b}$ is skew-self adjoint with respect to $g$. The argument following (3.8) applied to $h_{a b}=\lambda_{a b}$ then completes the proof of the first part of the lemma. Part $(i)$ of the lemma then also follows if $T_{m} M$ is an eigenspace of $\lambda_{a b}$ with respect to $g$. [It is remarked here that, for such a symmetric tensor, the eigenspaces corresponding to distinct eigenvalues are orthogonal.] For part (ii) one notes that, from part $(i), \lambda_{a b}=\sigma g_{a b}$ on $U$ for some smooth function $\sigma: U \rightarrow \mathbb{R}$. The Ricci identity on $\lambda$ then shows that

$$
\lambda_{d} R_{a b c}^{d}=\lambda_{a ; b c}-\lambda_{a ; c b}=g_{a b} \sigma_{, c}-g_{a c} \sigma_{, b},
$$

where a comma denotes a partial derivative. Now for $m^{\prime} \in U$ there exists $0 \neq F \in \operatorname{ker} \tilde{f}$ at $m^{\prime}$ and a contraction of (5.14) with $F^{b c}$ gives

$$
g_{a b}\left(F^{b c} \sigma_{, c}\right)-g_{a c}\left(F^{b c} \sigma_{, b}\right)=0
$$


from which it follows that $g_{a b} F^{b c} \sigma_{, c}=0$ and so $F^{a b} \sigma_{, b}=0$. So either $F$ is non-simple (and hence $d \sigma=0$ ) at $m^{\prime}$ or each member of ker $\tilde{f}$ is simple and the 1-form $d \sigma$ is $g$-orthogonal to its blade. In the latter case, if $\operatorname{dim}(\operatorname{ker} \tilde{f})=1$, and $\operatorname{ker} \tilde{f}$ is spanned by a single simple bivector the conditions of $(i)$ are not satisfied, whereas, if $\operatorname{dim}(\operatorname{ker} \tilde{f})=2$, ker $\tilde{f}$ must be spanned by two simple bivectors whose blades intersect in a 1-dimensional subspace of $T_{m^{\prime}} M$ and which again results in condition $(i)$ failing. If $\operatorname{dim}(\operatorname{ker} \tilde{f})=3$ it can be checked that either ker $\tilde{f}$ is spanned by three simple bivectors whose blades intersect in a 1-dimensional subspace of $T_{m^{\prime}} M$ (in which case $d \sigma$ is forced to be zero) or ker $\tilde{f}$ is spanned by three simple bivectors the blades of whose duals intersect in a 1-dimensional subspace of $T_{m^{\prime}} M$ (and $(i)$ fails). If $\operatorname{dim} \operatorname{ker} \tilde{f} \geq 4, \operatorname{ker} \tilde{f}$ must contain a non-simple member and $d \sigma=0$ must hold (see [2, p. 392]). It follows that $d \sigma \equiv 0$ on $U$ and, since $U$ is connected, the result $(i i)(a)$ follows. The result $(i i)(b)$ is then immediate from (5.14) and the result $(i i)(c)$ follows from (5.11).

It is remarked that, in the construction of examples, the concept of local projective relatedness will be required. For a space-time $(M, g)$ let $U$ be a non-empty connected open subset of $M$ and let $g^{\prime}$ be some metric defined on $U$. Then $g$ and $g^{\prime}$ (or their respective Levi-Civita connections) will be said to be (locally) projectively related (on $U$ ) if the restriction of $g$ to $U$ is projectively related to $g^{\prime}$ on $U$.

\section{Projective structure and holonomy I}

The relationship between holonomy type and projective relatedness can now be given. Amongst the holonomy types studied are several which include significant solutions to Einstein's field equations in general relativity (in addition to the vacuum solutions already discussed). For example, one has the non-vacuum pp-waves (type $R_{3}$ and $R_{8}$ ), the Gödel metric $\left(R_{10}\right)$, the Bertotti-Robinson metrics $\left(R_{7}\right)$ and the Einstein static universe $\left(R_{13}\right)$. For each holonomy type the general idea is, first, to determine the holonomy invariant distributions peculiar to that type and identify any covariantly constant or recurrent vector fields, second to link these vector fields to Riem using the infinitesimal holonomy structure, third to use Lemma 5.1 and Theorem 3.1 to find expressions for the 1-form $\lambda$ and the tensor $a$ and finally to use (5.7) to complete the procedure. It is convenient to break up the holonomy types into certain subcollections for easier handling. All metric and connection statements and index raising are understood to apply to the structures $g$ and $\nabla$ originally given on $M$. The following preliminary topological lemma, which is a generalised version of a result in [2], is useful in some of the theorems.

\section{Lemma 6.1.}

(i) Let $X$ be a topological space and let $A$ and $B$ be disjoint subsets of $X$ such that $A$ and $A \cup B$ are open in $X$ and $A \cup B$ is dense in $X$. Suppose $B=B_{1} \cup B_{2}$, with $B_{1}$ and $B_{2}$ disjoint, and int $B \subset$ int $B_{1} \cup B_{2}$ where int denotes the interior operator in the manifold topology of $M$. Then $X$ may be disjointly decomposed as $X=A \cup \operatorname{int} B_{1} \cup \operatorname{int} B_{2} \cup J$ where $J$ is the closed subset of $X$ defined by the disjointness of the decomposition and $A \cup \operatorname{int} B_{1} \cup \operatorname{int} B_{2}$ is open and dense in $X$ (that is, int $J=\varnothing)$.

(ii) Let $X$ be topological space and let $A_{1}, \ldots, A_{n}$ be disjoint subsets of $X$ such that $A_{1}$ together with $\cup_{i=1}^{i=k} A_{i}$ for $k=2, \ldots, n$ are open subsets of $X$ and such that $\cup_{i=1}^{i=n} A_{i}$ is (open and) dense in $X$. Then $X$ may be disjointly decomposed as $X=A_{1} \cup \operatorname{int} A_{2} \cup \cdots \cup \operatorname{int} A_{n} \cup K$ where $K$ is the closed subset of $X$ defined by the disjointness of the decomposition and $A_{1} \cup \operatorname{int} A_{2} \cup \cdots \cup \operatorname{int} A_{n}$ is open and dense in $X$ (that is, int $K=\varnothing$ ).

Proof. (i) Suppose int $J \neq \varnothing$ and let $U$ be a non-empty open subset of $X$ with $U \subset J$. Then $U \cap(A \cup B)$ is open and non-empty (since $A \cup B$ is dense in $M$ ) but $U$ is disjoint from 
$A$, int $B_{1}$ and $\operatorname{int} B_{2}$. Then $U \cap B$ is open and non-empty and hence so is $U \cap \operatorname{int} B$. But $\varnothing \neq U \cap \operatorname{int} B \subset U \cap\left(\right.$ int $\left.B_{1} \cup B_{2}\right)=U \cap B_{2}$ and so $U \cap B_{2}$ contains the non-empty, open subset $U \cap \operatorname{int} B$ of $M$ from which the contradiction that $U \cap \operatorname{int} B_{2} \neq \varnothing$ follows. Thus, $U=\varnothing$, $\operatorname{int} J=\varnothing$ and the result follows.

(ii) Suppose $U$ is an non-empty open subset of $X$ with $U \subset K$. Then $U \cap\left(\cup_{i=1}^{i=n} A_{i}\right)$ is not empty but $U$ is disjoint from $A_{1}, \operatorname{int} A_{2}, \ldots, \operatorname{int} A_{n}$. It follows that if the open set $U \cap\left(A_{1} \cup A_{2}\right)\left(=U \cap A_{2}\right)$ is non-empty, then $U \cap A_{2}$ is non-empty and open and gives the contradiction that $U \cap \operatorname{int} A_{2}$ is (open and) non-empty. Thus $U$ is disjoint from $A_{2}$. Continuing this sequence one finally gets the contradiction that $U$ is disjoint from each $A_{i}$ and hence from the open dense set $\cup_{i=1}^{i=n} A_{i}$. Thus $U=\varnothing$ and the result follows.

Theorem 6.1. Let $(M, g)$ and $\left(M, g^{\prime}\right)$ be space-times with $(M, g)$ non-flat. Suppose that $(M, g)$ is of holonomy type $R_{2}, R_{3}$ or $R_{4}$ and that $\nabla$ and $\nabla^{\prime}$ are projectively related. Then $\nabla=\nabla^{\prime}$ on $M$.

Proof. Suppose first that $(M, g)$ has holonomy type $R_{2}$ and, by the non-flat condition, let $U$ be the open dense subset of $M$ on which Riem is non-zero. Then, for $m \in U$ (see Section 4), there exists a connected and simply connected open neighbourhood $V \subset U$ of $m$ and two orthogonal smooth unit spacelike vector fields $X$ and $Y$ on $V$, spanning a holonomy invariant distribution at each point of $V$ and which are covariantly constant on $V$. The Ricci identity then reveals that $R_{b c d}^{a} X^{d}=R_{b c d}^{a} Y^{d}=0$ on $V$ and so Riem takes the curvature class $\mathbf{D}$ form (3.3) where $F$ is a smooth simple timelike bivector field on $V$ whose blade is orthogonal to the 2-spaces $X(m) \wedge Y(m)$ at each $m \in V$. Then at each $m \in V$ one may construct a null tetrad $l, n$, $x, y$, based on the holonomy invariant subspaces, so that $x=X(m)$ and $y=Y(m)$ and then $R_{a b c d} G^{c d}=0$ is satisfied by at $m$ for $G=l \wedge x, l \wedge y, n \wedge x, n \wedge y$ and $x \wedge y$. It follows that the conditions of Lemma 5.1 are satisfied on (some possibly reduced version of) $V$ and hence that the conclusions $(a),(b)$ and $(c)$ of this lemma hold. Then part $(c)$ of Lemma 5.1 and Theorem 3.1(i) show that, on $V$,

$$
a_{a b}=\phi g_{a b}+\mu X_{a} X_{b}+\nu Y_{a} Y_{b}+\rho\left(X_{a} Y_{b}+Y_{a} X_{b}\right)
$$

for functions $\phi, \mu, \nu$ and $\rho$. Also the smoothness of the functions $a_{a b} X^{a} X^{b}(=\phi+\mu), a_{a b} Y^{a} Y^{b}$ $(=\phi+\nu), a_{a b} X^{a} Y^{b}(=\rho)$ and $g^{a b} a_{a b}(=4 \phi+\mu+\nu)$ reveal the smoothness of the functions $\phi$, $\mu, \nu$ and $\rho$ on $V$. Now one substitutes (6.1) into (5.7) to get

$$
g_{a b} \phi_{, c}+X_{a} X_{b} \mu_{, c}+Y_{a} Y_{b} \nu_{, c}+\left(X_{a} Y_{b}+Y_{a} X_{b}\right) \rho_{, c}=g_{a c} \lambda_{b}+g_{b c} \lambda_{a} .
$$

A contraction of (6.2) successively with $l^{a} x^{b}$ and $n^{a} y^{b}$ at any $m \in V$ shows that $\lambda_{a} x^{a}=\lambda_{a} l^{a}=$ $\lambda_{a} y^{a}=\lambda_{a} n^{a}=0$. Thus the 1-form $\lambda$ is zero on $V$. It follows from (5.6) that the 1 -form $\psi$ is identically zero on $V$ and hence, from (5.1) that $\nabla=\nabla^{\prime}$ on $V$, hence on $U$ and thus on $M$. This completes the proof for holonomy type $R_{2}$ and the proofs for the holonomy types $R_{3}$ and $R_{4}$ are similar; for type $R_{3}$ one has covariantly constant, orthogonal, null and spacelike vector fields $L$ and $Y$, respectively, on $V$ and the holonomy invariant distributions can be used construct vector fields $N$ and $X$ on (a possibly reduced) $V$ such that $L, N, X$ and $Y$ give a null tetrad at each point of $V$ from which the proof follows in a similar way to that of the $R_{2}$ type. For type $R_{4}$ one has covariantly constant null vector fields $L$ and $N$ on $V$ which one can choose to satisfy $g_{a b} L^{a} N^{b}=1$. Again one easily achieves $\nabla=\nabla^{\prime}$ on $M$.

Theorem 6.2. Let $(M, g)$ and $\left(M, g^{\prime}\right)$ be space-times with $(M, g)$ non-flat and of holonomy type $R_{7}$. Suppose that $\nabla$ and $\nabla^{\prime}$ are projectively related. Then $\nabla=\nabla^{\prime}$ on $M$.

Proof. Let $U$ be the open dense subset of $M$ on which Riem does not vanish. If $m \in U$ there exists an open, connected and simply connected neighbourhood $V \subset U$ and null properly 
recurrent vector fields $L$ and $N$ scaled so that $L^{a} N_{a}=1$ on $V$ (noting that this scaling will not affect their recurrence property). Then $\nabla L=L \otimes P$ and $\nabla N=-N \otimes P$ for some smooth 1-form field $P$ on $V$. The simple bivector field $F \equiv L \wedge N,\left(F_{a b} \equiv 2 L_{[a} N_{b]}\right)$, then satisfies $\nabla F=0$ and its (simple) dual bivector field also satisfies $\nabla \stackrel{*}{F}=0$. The blades of $F$ and $\stackrel{*}{F}$ span, respectively, the timelike and spacelike holonomy distributions on $V$ and reducing $V$, if necessary, one may write $\stackrel{*}{F}_{a b}=2 X_{a[} Y_{b]}$ for smooth unit orthogonal spacelike vector fields $X$ and $Y$ on $V$ and which satisfy $X^{a} X_{a ; b}=Y^{a} Y_{a ; b}=0$. The condition $\nabla \stackrel{*}{F}=0$ on $V$ shows that $\nabla X=Y \otimes Q$ and $\nabla Y=-X \otimes Q$ on $V$ for some smooth 1-form $Q$ on $V$. At each $m \in V, L(m)$, $N(m), X(m)$ and $Y(m)$ give a null tetrad $l, n, x, y$ at $m$. Now at each $m \in U$, Table 1 and a consideration of the infinitesimal holonomy structure of $(M, g)$ show that the curvature class of Riem at $m$ is either $\mathbf{D}$ or $\mathbf{B}$, taking the form (3.2) if it is $\mathbf{B}$ and (3.3) with $F$ either timelike or spacelike if it is $\mathbf{D}$. Let $\mathbf{B}, \mathbf{D}_{\mathbf{s}}$ and $\mathbf{D}_{\mathbf{t}}$ denote the subsets of $U$ consisting of those points at which the curvature class is, respectively, $\mathbf{B}, \mathbf{D}$ with $F$ spacelike or $\mathbf{D}$ with $F$ timelike. Then one may decompose $M$, disjointly, as $M=\mathbf{B} \cup \mathbf{D}_{\mathbf{s}} \cup \mathbf{D}_{\mathbf{t}} \cup J=\mathbf{B} \cup \operatorname{int} \mathbf{D}_{\mathbf{s}} \cup \operatorname{int} \mathbf{D}_{\mathbf{t}} \cup K$ where, by the "rank" theorem (see e.g. [2]), B is open in $M$ and $J$ and $K$ are closed subsets of $M$ defined by the disjointness of the decomposition and $J$ satisfies int $J=\varnothing$. If $\operatorname{int} \mathbf{D}_{\mathbf{s}} \neq \varnothing$ let $m \in \operatorname{int} \mathbf{D}_{\mathbf{s}}$ and $m \in V \subset \operatorname{int} \mathbf{D}_{\text {s }}$ with $V$ as above. Then (3.3) holds on $V$ with $F=X \wedge Y$ and $\alpha$ smooth on $V$. It is then clear that, at $m, l \wedge x, l \wedge y, n \wedge x, n \wedge y$ and $l \wedge n$ are in ker $\tilde{f}$ and so, from Lemma $5.1(i i)(a), T_{m} M$ is an eigenspace of $\nabla \lambda$ and so $\nabla \lambda=c g$ on $V$. Then Lemma 5.1(ii)(c) and Theorem $3.1(i)$ show that

$$
a_{a b}=\phi g_{a b}+\mu L_{a} L_{b}+\nu N_{a} N_{b}+\rho\left(L_{a} N_{b}+N_{a} L_{b}\right)
$$

for functions $\phi, \mu, \nu$, and $\rho$ on $V$ which are smooth since $a_{a b} L^{a} L^{b}, a_{a b} N^{a} N^{b}, a_{a b} L^{a} N^{b}$ and $g^{a b} a_{a b}$ are. Noting that $L_{(a} N_{b)}$ is covariantly constant on $V$, a substitution of (6.3) into (5.7) gives, on $V$,

$$
\begin{aligned}
& g_{a b} \phi_{, c}+L_{a} L_{b} \mu_{, c}+2 \mu L_{a} L_{b} P_{c}+N_{a} N_{b} \nu_{, c}-2 \nu N_{a} N_{b} P_{c}+\left(L_{a} N_{b}+N_{a} L_{b}\right) \rho_{, c} \\
& \quad=g_{a c} \lambda_{b}+g_{b c} \lambda_{a} .
\end{aligned}
$$

Successive contractions of (6.4) with $L^{a} X^{b}$ and $N^{a} Y^{b}$ then show that $\lambda_{a} L^{a}=\lambda_{a} N^{a}=\lambda_{a} X^{a}=$ $\lambda_{a} Y^{a}=0$ on $V$. Thus $\lambda$ vanishes on $V$ and so on $\operatorname{int} \mathbf{D}_{\mathbf{s}}$. If int $\mathbf{D}_{\mathbf{t}} \neq \varnothing$ a similar argument using (3.3) with $F=L \wedge N$ can be used to show that $\lambda$ vanishes on $\operatorname{int} \mathbf{D}_{\mathbf{t}}$. If $\mathbf{B} \neq \varnothing$, Lemma 5.1(ii)(b) shows that $\lambda$ vanishes on $\mathbf{B}$ since no non-trivial solutions for $\lambda$ of the equation displayed there exist at points of curvature class $\mathbf{B}$. So one has achieved the situation that $M=\mathbf{B} \cup \mathbf{D} \cup J$ with int $J=\varnothing$ and $\mathbf{B}$ and $\mathbf{B} \cup \mathbf{D}$ open in $M$ (again by an application of the "rank" theorem) and with $\lambda$ vanishing on the open subset $\mathbf{B} \cup \operatorname{int} \mathbf{D}_{\mathbf{s}} \cup \operatorname{int} \mathbf{D}_{\mathbf{t}}$. That this open subset is a dense subset of $M$ follows from Lemma 6.1(i) by the following argument. Since $\mathbf{D} \equiv \mathbf{D}_{\mathbf{s}} \cup \mathbf{D}_{\mathbf{t}}$ then, from elementary topology, int $\mathbf{D}_{\mathbf{s}} \cup \operatorname{int} \mathbf{D}_{\mathbf{t}} \subset \operatorname{int} \mathbf{D}$. That the reverse inclusion is true in this case follows from noting that it is trivially true if int $\mathbf{D}=\varnothing$ whilst if $\operatorname{int} \mathbf{D} \neq \varnothing$ one writes equation (3.3) for Riem on some open neighbourhood of any point $m$ in the submanifold int $\mathbf{D}$ with $\alpha$ and $F$ smooth (as one can) and then defines the smooth map $\theta$ from this neighbourhood to $\mathbb{R}$ by $\theta(m)=\left(F^{a b} F_{a b}\right)(m)$ (so that $\theta(m)>0$ if $m \in \mathbf{D}_{\mathbf{s}}$ and $\theta(m)<0$ if $\left.m \in \mathbf{D}_{\mathbf{t}}\right)$. It is easily seen now that $m$ is either in $\operatorname{int} \mathbf{D}_{\mathbf{s}}$ or $\operatorname{int} \mathbf{D}_{\mathbf{t}}$. Thus $\operatorname{int} \mathbf{D}=\operatorname{int} \mathbf{D}_{\mathbf{s}} \cup \operatorname{int} \mathbf{D}_{\mathbf{t}}$ and the conditions of Lemma 6.1(i) are satisfied. So $\lambda$ vanishes on an open dense subset of $M$ and hence on $M$. It follows that $\nabla=\nabla^{\prime}$ on $M$.

Theorem 6.3. Let $(M, g)$ and $\left(M, g^{\prime}\right)$ be space-times with $(M, g)$ non-flat and of holonomy type $R_{10}, R_{11}$ or $R_{13}$ and with Riem of curvature class $\mathbf{C}$ at each point of an open, dense subset $U$ of $M$. Suppose that $\nabla$ and $\nabla^{\prime}$ are projectively related. Then $\nabla=\nabla^{\prime}$ on $M$. 
Proof. Suppose that $(M, g)$ is of holonomy type $R_{10}$ (the arguments for types $R_{11}$ and $R_{13}$ are similar). For any $m \in U$ there exists an open, connected and simply connected neighbourhood $V \subset U$ of $m$ and a unit spacelike covariantly constant vector field $X$ on $V$. Then one may choose an orthonormal tetrad $u, x, y, z$ at $m$ such that $X(m)=x$ and, from the Ricci identity on $X$, $R_{b c d}^{a} X^{d}=0$ on $V$, and so $x \wedge u, x \wedge y$ and $x \wedge z$ are in ker $\tilde{f}$ at $m$. As before, one sees that the conditions and conclusions of Lemma 5.1 hold at each point of $V$ and so Theorem 3.1(ii) gives

$$
a_{a b}=\phi g_{a b}+\mu X_{a} X_{b}
$$

on $V$ for functions $\phi$ and $\mu$ which are smooth since $a_{a b} X^{a} X^{b}$ and $g^{a b} a_{a b}$ are. On substituting (6.5) into (5.7) one finds

$$
g_{a b} \phi_{, c}+X_{a} X_{b} \mu_{, c}=g_{a c} \lambda_{b}+g_{b c} \lambda_{a}
$$

and successive contractions of (6.6) with $x^{a} y^{b}$ and $u^{a} z^{b}$ show that $\lambda$ vanishes at $m$, hence on $V$ and so on $M$. It follows that $\nabla=\nabla^{\prime}$ on $M$.

Theorem 6.4. Let $(M, g)$ and $\left(M, g^{\prime}\right)$ be space-times with $(M, g)$ non-flat and of holonomy type $R_{6}, R_{8}$ or $R_{12}$. Suppose that $\nabla$ and $\nabla^{\prime}$ are projectively related. Then $\nabla=\nabla^{\prime}$ on $M$.

Proof. In each case let $U$ be the open dense subset of $M$ on which Riem does not vanish. Then any $m \in U$ admits an open, connected and simply connected neighbourhood $V \subset U$ on which are defined orthogonal vector fields $L, X$ and $Y$, with $L$ null and either recurrent or covariantly constant (depending on the holonomy type) and $X$ and $Y$ unit spacelike vector fields and which together span a 3-dimensional null holonomy invariant distribution on $V$. By reducing $V$, if necessary, one may assume the existence of a null vector field $N$ which along with $L, X$ and $Y$ span a null tetrad at each point of $V$.

Now for holonomy type $R_{6}$ one may take $L$ recurrent and $Y$ covariantly constant. The curvature class at $m$ is either $\mathbf{C}$ or $\mathbf{D}$ and if it is $\mathbf{D}$, equation (3.3) holds with $F=l \wedge x$ or $F=l \wedge n+a l \wedge x(a \in \mathbb{R})$. Let the subset of points of $M$ at which the first of these class $\mathbf{D}$ possibilities holds be denoted by $\mathbf{D}_{\mathbf{n}}$ (null) and at which the second holds by $\mathbf{D}_{\mathbf{n n}}$ (non-null). Then let $\mathbf{D} \equiv \mathbf{D}_{\mathbf{n}} \cup \mathbf{D}_{\mathbf{n n}}$. Let the subset of points of $M$ at which at which the curvature class is $\mathbf{C}$ be denoted by $\mathbf{C}$. Then $\mathbf{C}$ is open in $M$ and $U=\mathbf{C} \cup \mathbf{D}$. $M$ admits the disjoint decomposition $M=\mathbf{C} \cup \mathbf{D}_{\mathbf{n}} \cup \mathbf{D}_{\mathbf{n n}} \cup J$ where $J$ is the closed subset of $M$ determined by the disjointness of the decomposition and int $J=\varnothing$. It is clear that, whatever the curvature type, the conditions and conclusions of Lemma 5.1 hold. If $\mathbf{C} \neq \varnothing$ and $m \in \mathbf{C}$ then one may arrange in the previous paragraph that $m \in V \subset U$ and that, on $V$,

$$
a_{a b}=\phi g_{a b}+\mu Y_{a} Y_{b}
$$

for functions $\phi$ and $\mu$. The proof now proceeds as for Theorem 6.3 and one obtains $\nabla=\nabla^{\prime}$ on $\mathbf{C}$.

If int $\mathbf{D}_{\mathbf{n}} \neq \varnothing$ then with $V$ as above and $m \in V \subset \operatorname{int} \mathbf{D}_{\mathbf{n}}$, Lemma 5.1 and Theorem 3.1( $\left.i\right)$ give, on $V$,

$$
a_{a b}=\phi g_{a b}+\mu L_{a} L_{b}+\nu Y_{a} Y_{b}+\rho\left(L_{a} Y_{b}+Y_{a} L_{b}\right)
$$

for functions $\phi, \mu, \nu$ and $\rho$ which are smooth since $a_{a b} X^{a} X^{b}, a_{a b} Y^{a} Y^{b}, a_{a b} N^{a} N^{b}$ and $a_{a b} N^{a} Y^{b}$ are. On substituting (6.8) into (5.7) and contracting, successively, with $X^{a} Y^{b}, L^{a} L^{b}$ and $N^{a} X^{b}$ one again finds that $\lambda=0$ on $V$ and hence on $\operatorname{int}_{\mathbf{n}}$ and so $\nabla=\nabla^{\prime}$ on int $\mathbf{D}_{\mathbf{n}}$. (This argument includes within it the curvature class $\mathbf{C}$ case above; one simply sets $\mu$ and $\rho$ to zero in (6.8).)

If int $\mathbf{D}_{\mathbf{n n}} \neq \varnothing$ and $m \in V \subset \operatorname{int} \mathbf{D}_{\text {nn }}$ with $V$ as above, Riem takes the form (3.3) with $F$ chosen as $F=L \wedge N+\sigma L \wedge X$ for a smooth function $\sigma$, on $V$. Now define smooth vector fields 
$X^{\prime}=X-\sigma L$ and $N^{\prime}=N+\sigma X-\frac{\sigma^{2}}{2} L$ on $V$, noting that $L, N^{\prime}, X^{\prime}$ and $Y$ still give rise to a null tetrad in the obvious way at each point of $V$ and that now $F=L \wedge N^{\prime}$ on $V$. Again Lemma 5.1 applies and Theorem 3.1(i) gives

$$
a_{a b}=\phi g_{a b}+\mu X_{a}^{\prime} X_{b}^{\prime}+\nu Y_{a} Y_{b}+\rho\left(X_{a}^{\prime} Y_{b}+Y_{a} X_{b}^{\prime}\right),
$$

where, as before, $\phi, \mu, \nu$ and $\rho$ are smooth functions on $V$. On substituting (6.9) into (5.7) (and noting that, since $L$ is recurrent, $\left.L^{a} X_{a ; b}^{\prime}=0\right)$ successive contractions with $N^{\prime a} N^{\prime b}, L^{a} X^{\prime b}$ and $L^{a} Y^{b}$ again reveal that $\nabla=\nabla^{\prime}$ on int $\mathbf{D}_{\mathbf{n n}}$. So $\nabla=\nabla^{\prime}$ on $\tilde{W} \equiv \mathbf{C} \cup \operatorname{int} \mathbf{D}_{\mathbf{n}} \cup \operatorname{int} \mathbf{D}_{\mathbf{n n}}$. That the open set $\tilde{W}$ is dense in $M$ follows from Lemma $6.1(i)$. To see this let $K \equiv M \backslash \tilde{W}$ and let $W$ be a non-empty open subset of $K$. Then $W$ is disjoint from $\mathbf{C}$, int $\mathbf{D}_{\mathbf{n}}$ and int $\mathbf{D}_{\mathbf{n n}}$ but the open subset $W \cap(\mathbf{C} \cup \mathbf{D})=W \cap U \neq \varnothing$ since $U$ is dense in $M$. It follows that $W \cap \mathbf{D}$ and hence $W \cap$ intD are open and non-empty. Now, as in the previous theorem, consider the map arising from the bivector $F$ in (3.3) on the (non-empty) open submanifold intD (and note that for points in $\mathbf{D}_{\mathbf{n}}$ (where $F$ null) its value is zero whereas for points in $\mathbf{D}_{\mathbf{n n}}$ its value is negative). A consideration of the open subset of intD given by the inverse image, under $f$, of the negative reals shows that int $\mathbf{D} \subset \operatorname{int} \mathbf{D}_{\mathbf{n n}} \cup \mathbf{D}_{\mathbf{n}}$. The result now follows from Lemma $6.1(i)$ and $\lambda$ vanishes on $\tilde{W}$ and hence on $M$. It follows that $\nabla=\nabla^{\prime}$ and this completes the proof when the holonomy type is $R_{6}$.

If $(M, g)$ has holonomy type $R_{8}$ and if $m \in U$ the curvature class of Riem at $m$ is either $\mathbf{D}$ (equation (3.3) with $F$ null) or C. A neighbourhood $V$ and vector fields $L, N, X$ and $Y$, can be established, as in the above paragraphs, with $L$ covariantly constant. Lemma 1 again applies and Theorem 3.1, parts $(i)$ and (ii), then lead to a straightforward proof that $\lambda=0$ on (and in an obvious notation) the open subset $\mathbf{C}$ and on int $\mathbf{D}$. (For class $\mathbf{D}$, useful contractions of (5.7) are with $L^{a} L^{b}, L^{a} X^{b}, L^{a} Y^{b}, Y^{a} Y^{b}$ and $L^{a} N^{b}$ and in the class $\mathbf{C}$ case, with $L^{a} X^{b}, L^{a} Y^{b}$ and $X^{a} N^{b}$.) That the open subset $\mathbf{C} \cup \operatorname{int} \mathbf{D}$ is dense in $M$ then follows from Lemma 6.1(ii) since $\mathbf{C} \cup \mathbf{D}$ is open and dense in $M$. Thus $\lambda=0$ and $\nabla=\nabla^{\prime}$ on $M$.

If $(M, g)$ has holonomy type $R_{12}$ then for $m \in U$ one may choose a neighbourhood $V$ of $m$ and vector fields $L, N, X$ and $Y$ as above with the null vector field $L$ recurrent. Table 1 shows that $l \wedge x, l \wedge y$ and $l \wedge n+\omega^{-1} x \wedge y$ lie in ker $\tilde{f}$ and so Lemma 5.1 again applies. If $m \in U$ the possible curvature classes for Riem at $m$ are $\mathbf{D}$ with $F$ null in (3.3) (since the only linear combinations of the members of the bivector algebra for type $R_{12}$ which are simple are null), $\mathbf{C}$ (with $R_{b c d}^{a} l^{d}=0$ at $m$ ) or $\mathbf{A}$. One then decomposes $M^{\prime}$, in an obvious notation, as $M^{\prime}=\mathbf{A} \cup \operatorname{int} \mathbf{C} \cup \operatorname{int} \mathbf{D} \cup J$ (recalling from Section 3 that $\mathbf{A}$ is an open subset of $M$ ). The conclusion (b) of Lemma 5.1(ii) then immediately shows that $\lambda=0$ on $\mathbf{A}$ and procedures similar to those already given reveal that $\lambda=0$ on int $\mathbf{C}$ and int $\mathbf{D}$ (for the latter contract (5.7) with $L^{a} L^{b}, L^{a} X^{b}, L^{a} Y^{b}, Y^{a} Y^{b}$ and $\left.N^{a} L^{b}\right)$. Since $\mathbf{A}, \mathbf{A} \cup \mathbf{C}$ and $\mathbf{A} \cup \mathbf{C} \cup \mathbf{D}$ are open in $M$, with the latter dense, Lemma 6.1(ii) completes the proof that $\lambda=0$ and $\nabla=\nabla^{\prime}$ on $M$.

In summary, if $(M, g)$ is non-flat and if, in addition, it is either of holonomy type $R_{2}, R_{3}$, $R_{4}, R_{6}, R_{7}, R_{8}$ or $R_{12}$ or of holonomy type $R_{10}, R_{11}$ or $R_{13}$ and of curvature class $\mathbf{C}$ over some open dense subset of $M$ then if $g^{\prime}$ is another metric on $M$ projectively related to $g, \nabla=\nabla^{\prime}$ on $M$. One can say a little more here because in each of the above holonomy types (including the curvature class $\mathbf{C}$ clause applied to the types $R_{10}, R_{11}$ and $\left.R_{13}\right)$ the condition that $\nabla$ and $\nabla^{\prime}$ are projectively related leads to $\nabla=\nabla^{\prime}$ and hence to the condition that the holonomy groups of $(M, g)$ and $\left(M, g^{\prime}\right)$ are the same. It is then easy to write down a simple relation between the metrics $g$ and $g^{\prime}[15,2]$. For example, suppose either that $M$ is simply connected or that one is working over a connected and simply connected open region $V$ of $M$ (on which the holonomy type is the same as that of $M$ ). Then if this common holonomy type is $R_{2}, R_{3}$ or $R_{4}$, one gets a relation of the form

$$
g_{a b}^{\prime}=\phi g_{a b}+\mu p_{a} p_{b}+\nu q_{a} q_{b}+\rho\left(p_{a} q_{b}+q_{a} p_{b}\right),
$$


where $\phi, \mu, \nu$ and $\rho$ are constants (subject only to a non-degeneracy condition and signature requirements) and where $p$ and $q$ are vector fields on $M$ (or $V$ ) spanning the 2-dimensional vector space of covariantly constant vector fields. For holonomy types $R_{6}$ and $R_{8}$ or types $R_{10}$, $R_{11}$ or $R_{13}$ (with the above clauses attached) one gets

$$
g_{a b}^{\prime}=\phi g_{a b}+\mu k_{a} k_{b}
$$

where $\phi$ and $\mu$ are constants (again subject to non-degeneracy and signature requirements) and $k$ is a vector field on $M$ (or $V$ ) spanning the vector space of covariantly constant vector fields. For holonomy type $R_{7}$ one gets

$$
g_{a b}^{\prime}=\phi g_{a b}+\mu\left(l_{a} n_{b}+n_{a} l_{b}\right),
$$

where again $\phi$ and $\mu$ are constants (again subject to non-degeneracy and signature requirements) and $l$ and $n$ are null, properly recurrent vector fields on $M$ (or $V$ ). For holonomy type $R_{12}$ one gets $g_{a b}^{\prime}=\phi g_{a b}$ with $\phi$ constant. [It is remarked here that for holonomy types $R_{10}, R_{11}$ or $R_{13}$, even without the curvature class $\mathbf{C}$ clause, if it is given that $\nabla=\nabla^{\prime}$ then (6.11) follows for constant $\phi$ and $\mu$. But, in this latter case, projective relatedness without this clause does not force the condition $\nabla=\nabla^{\prime}$ as will be seen later.] It is clear from these results that if $g$ and $g^{\prime}$ are projectively related metrics they may have different signatures.

\section{$7 \quad$ Projective structure and holonomy II}

In this section the holonomy types (and special cases of holonomy types) omitted in the last section will be discussed. Theorem 6.3 dealt in detail with the projective relatedness problem for the (non-flat) holonomy types $R_{10}, R_{11}$ and $R_{13}$ when the curvature rank of the map $f$ in (3.1) (and which, for each of these types, is necessarily at most 3 ) is equal to 2 or 3 (curvature class $\mathbf{C}$ ) at each point of an open, dense subset of $M$. One can now consider the situation for these holonomy types when the curvature rank is 1 (curvature class $\mathbf{D}$ ) at each point of some open, dense subset $U$ of $M$. For any of these holonomy types define subsets $M_{1} \equiv\{m \in M: \lambda$ vanishes on some open neighbourhood of $m\}$ and $M_{2} \equiv\{m \in M: \lambda$ does not vanish on any open neighbourhood of $m\}=M \backslash M_{1}$. Then one has the disjoint decomposition of $M$ given by $M=M_{1} \cup M_{2}$ with $M_{1}$ open in $M$. Let $J=M \backslash U$ so that $J$ is closed in $M$ and int $J=\varnothing$ (since $U$ is open and dense in $M$ ). Next, if $\mathbf{D}_{\mathbf{n n}}$ is the subset of points of $\mathrm{M}$ at which the bivector $F$ in (3.3) is either spacelike or timelike and $\mathbf{D}_{\mathbf{n}}$ the subset of points where it is null then $U=\mathbf{D}_{\mathbf{n n}} \cup \mathbf{D}_{\mathbf{n}}$ with $\mathbf{D}_{\mathbf{n n}}$ an open subset of $M$ (by a consideration of the map associated with the smooth function $F_{a b} F^{a b}$ on $U$ and described earlier). One then gets a disjoint decomposition $M=M_{1} \cup\left(M_{2} \cap \mathbf{D}_{\mathbf{n n}}\right) \cup \operatorname{int}\left(M_{2} \cap \mathbf{D}_{\mathbf{n}}\right) \cup K$. It will be seen, as the proof progresses, that in all cases $M_{2} \cap \mathbf{D}_{\mathbf{n n}}$ is also open in $M$ and hence $K$, which is defined by the disjointness of the decomposition, is closed in $M$. It then follows that, with the openness of $M_{2} \cap \mathbf{D}_{\mathbf{n n}}$ assumed, if $W$ is a non-empty open subset of $K, W \cap M_{1}=W \cap\left(M_{2} \cap \mathbf{D}_{\mathbf{n n}}\right)=W \cap \operatorname{int}\left(M_{2} \cap \mathbf{D}_{\mathbf{n}}\right)=\varnothing$. But the non-empty open set $W \cap U=W \cap\left(\mathbf{D}_{\mathbf{n}} \cup \mathbf{D}_{\mathbf{n n}}\right)=W \cap \mathbf{D}_{\mathbf{n}}$ (since $W \subset K$ and $\left.K \cap \mathbf{D}_{\mathbf{n n}}=\varnothing\right)=W \cap\left(M_{2} \cap \mathbf{D}_{\mathbf{n}}\right)$. Hence $W \cap \operatorname{int}\left(M_{2} \cap \mathbf{D}_{\mathbf{n}}\right) \neq \varnothing$ and this contradiction shows that int $K=\varnothing$. Thus, considering the open dense subset $M_{1} \cup\left(M_{2} \cap \mathbf{D}_{\mathbf{n n}}\right) \cup \operatorname{int}\left(M_{2} \cap \mathbf{D}_{\mathbf{n}}\right)$ of $\mathrm{M}$ one sees that since $\lambda=0$ on $M_{1}$ the interesting parts of $M$ in this context are the open subsets $M_{2} \cap \mathbf{D}_{\mathbf{n n}}$ and $\operatorname{int}\left(M_{2} \cap \mathbf{D}_{\mathbf{n}}\right)$ (and, in fact, $\mathbf{D}_{\mathbf{n}}=\varnothing$ in the $R_{13}$ case). In fact, it will turn out that, in all cases, int $\left(M_{2} \cap \mathbf{D}_{\mathbf{n}}\right)=\varnothing$.

So let $(M, g)$ be a (non-flat) space-time of holonomy type $R_{10}, R_{11}$ or $R_{13}$ whose curvature is of class $\mathbf{D}$ on some open dense subset $U$ of $M$. Thus (3.3) holds on $U$ (and Riem vanishes on $M \backslash U)$. Let $m \in U$ and let $V \subset U$ be a connected, simply connected, open neighbourhood of $m$ on which is defined a metric $g^{\prime}$, (locally) projectively related to $g$ on $V$, together with 
an associated pair $(a, \lambda)$. The conditions imposed on $(M, g)$ together with (3.3) show that the conditions and hence the conclusions of Lemma 5.1 hold on $U$ and hence on $V$. Thus $\nabla \lambda=c g$ holds on $V$ for some constant $c$.

Now suppose that the holonomy type of $(M, g)$ is $R_{11}$. Then $V$ can be chosen so that it admits a covariantly constant, null vector field $l$, so that, from the Ricci identity, $R_{b c d}^{a} l^{d}=0$. Also, from the curvature class $\mathbf{D}$ condition, $V$ (after a possible reduction, if necessary) admits another (smooth) nowhere-zero vector field $p$ such that $l\left(m^{\prime}\right)$ and $p\left(m^{\prime}\right)$ are independent members of $T_{m^{\prime}} M$ for each $m^{\prime} \in V$ and such that $R_{b c d}^{a} p^{d}=0$ holds on $V$. Condition $(b)$ of Lemma 5.1 and the curvature class $\mathbf{D}$ condition then show that, on $V$,

$$
\lambda_{a}=\sigma l_{a}+\rho p_{a}
$$

and so, since $\lambda_{a ; b}=c g_{a b}$, one gets

$$
\rho p_{a ; b}+p_{a} \rho_{, b}+l_{a} \sigma_{, b}=c g_{a b} .
$$

Also, part $(c)$ of Lemma 5.1 and Theorem 3.1( $i)$ give

$$
a_{a b}=\phi g_{a b}+\beta l_{a} l_{b}+\gamma p_{a} p_{b}+\delta\left(l_{a} p_{b}+p_{a} l_{b}\right)
$$

for smooth functions $\phi, \beta, \gamma$ and $\delta$ on $V$. Now there exists a nowhere-null, nowhere-zero vector field $q$ on (a possibly reduced) $V$ which is orthogonal to $l$ and $p$ and is such that $q_{a} q^{a}$ is constant (and hence $q^{a} q_{a ; b}=0$ ) on $V$. Then from (7.3), $a_{a b} q^{b}=\phi q_{a}$ on $V$. On covariantly differentiating this last equation and using (5.7) after a contraction with $q^{a}$ one finds that $\phi_{, a}=0$ and so $\phi$ is constant on $V$ (and non-zero since $a$ is non-degenerate).

Now the simple bivector $F$ in (3.3) satisfies $F_{a b} l^{b}=0$ and so is either null or spacelike at $m \in U$, that is, either $m \in \mathbf{D}_{\mathbf{n}}$ or $m \in \mathbf{D}_{\mathbf{n n}}$. Recalling the decomposition of $M$ given above assume $\operatorname{int}\left(M_{2} \cap \mathbf{D}_{\mathbf{n}}\right)$ is not empty and let $m \in V \subset \operatorname{int}\left(M_{2} \cap \mathbf{D}_{\mathbf{n}}\right)$ for (some possibly reduced) open neighbourhood $V$ as before. Then $p$ may be chosen a unit spacelike vector field on $V$ orthogonal to $l$ on $V$ and (7.3) shows that $a_{a b} l^{b}=\phi l_{a}$ which, after differentiation and making use of (5.7) and the constancy of $\phi$ and $l$ immediately shows that $\lambda$ vanishes on $V$. This contradicts the fact that $m \in M_{2}$. Thus, $\operatorname{int}\left(M_{2} \cap \mathbf{D}_{\mathbf{n}}\right)$ is empty.

If $F$ is spacelike at $m$, that is, $m \in \mathbf{D}_{\mathbf{n n}}$, and assuming that $M_{2} \cap \mathbf{D}_{\mathbf{n n}}$ is not empty let $m \in\left(M_{2} \cap \mathbf{D}_{\mathbf{n n}}\right)$ and choose $V$ such that $m \in V \subset \mathbf{D}_{\mathbf{n n}}$ (since $\mathbf{D}_{\mathbf{n n}}$ is open in $M$ ). Then one may choose $p$ so that $p$ is null and $l^{a} p_{a}=1$ on $V$. Then (7.2) on contraction first with $l^{a}$ and then with $p^{a}$ (using $p^{a} p_{a ; b}=0$ since $p$ is null on $V$ ) yields

$$
c l_{a}=\rho_{, a}, \quad c p_{a}=\sigma_{, a}
$$

and then (7.2) becomes

$$
\rho p_{a ; b}=c T_{a b}, \quad\left(T_{a b} \equiv g_{a b}-l_{a} p_{b}-p_{a} l_{b}\right) .
$$

Next, differentiate (7.3) and use (7.1) and (5.7) to get

$$
\begin{aligned}
& g_{a c}\left(\sigma l_{b}+\rho p_{b}\right)+g_{b c}\left(\sigma l_{a}+\rho p_{a}\right) \\
& \quad=l_{a} l_{b} \beta_{, c}+p_{a} p_{b} \gamma_{, c}+\gamma p_{a ; c} p_{b}+\gamma p_{a} p_{b ; c}+\left(l_{a} p_{b}+p_{a} l_{b}\right) \delta_{, c}+\delta\left(l_{a} p_{b ; c}+p_{a ; c} l_{b}\right) .
\end{aligned}
$$

On contracting this successively with $l^{a} l^{b}, p^{a} p^{b}$ and $l^{a} p^{b}$ (using $l^{a} p_{a ; b}=0$ ) one gets

$$
2 \rho l_{a}=\gamma_{, a}, \quad 2 \sigma p_{a}=\beta_{, a}, \quad \sigma l_{a}+\rho p_{a}=\delta_{, a} .
$$

If one finally contracts (7.6) first with $l^{a}$ and then with $p^{a}$ and use is made of (7.7) one easily finds

$$
\gamma p_{a ; b}=\rho T_{a b}, \quad \delta p_{a ; b}=\sigma T_{a b} .
$$


The condition $m \in M_{2}$ implies that $p_{a ; b}(m) \neq 0$ because, otherwise, (7.5) shows that $c=0$ and then (7.4) reveals that $\rho$ and $\sigma$ are constant on $V$. Then (7.8) shows that $\rho$ and $\sigma$ vanish at $m$ and hence on $V$. Finally, (7.1) implies that $\lambda$ vanishes on $V$ and a contradiction follows. It thus follows that $p_{a ; b}$ does not vanish at $m$ and hence on some open neighbourhood of $m$ (taken to be $V)$. Further, if $\lambda(m)=0$ then (7.1) shows that $\sigma(m)=\rho(m)=0$ and so, from (7.5), $c=0$. Then (7.4) reveals that $\sigma$ and $\rho$ are constant and hence zero on $V$ and hence, from (7.1), the contradiction that $\lambda \equiv 0$ on $V$. Thus $\lambda(m) \neq 0$ and so one may assume that $V$ is chosen (after a possible reduction) so that $\lambda$ and $p_{a ; b}$ are nowhere zero on $V$. But this implies that $V \subset M_{2}$ and hence that $V \subset\left(M_{2} \cap \mathbf{D}_{\mathbf{n n}}\right)$. From this it follows that $M_{2} \cap \mathbf{D}_{\mathbf{n n}}$ is open in $M$ (as remarked above).

Continuing with this case, if $p_{a ; b}$ is not a multiple of $T_{a b}$ at some $m^{\prime} \in V$ then (7.8) shows, since $T\left(m^{\prime}\right) \neq 0$, that $\rho\left(m^{\prime}\right)=\sigma\left(m^{\prime}\right)=0$ and then (7.1) gives the contradiction that $\lambda\left(m^{\prime}\right)=0$. So $p_{a ; b}$ is a non-zero multiple of $T_{a b}$ at each point of $V$. It follows that the covector fields associated with both $l$ and $p$ are closed 1-forms on $V$ and hence are gradients on (a possibly reduced) $V$, say of the (smooth) functions $u$ and $v$, respectively. In addition, $l$ and $p$ are easily checked to have zero Lie bracket and so span an integrable distribution (again on a possibly reduced $V$ ). It can then be shown by using standard techniques that if one chooses $x$ and $y$ coordinates on that part of the 2-dimensional submanifold $u=v=0$ contained in (a possibly reduced) $V$ and chooses the other coordinates as parameters along the integral curves of $l$ and $p$ (with $x$ and $y$ constant along these curves) the parameter coordinates may be taken as $u$ and $v$ and $l=\partial / \partial v$ and $p=\partial / \partial u$. Then with the coordinates $u, v, x, y$ the metric $g$ takes the form

$$
d s^{2}=2 d u d v+g_{\alpha \beta} d x^{\alpha} d x^{\beta} \quad\left(g_{\alpha \beta}=T_{\alpha \beta}\right),
$$

where Greek indices take the values 3 and 4 and the second (bracketed) equation in (7.5) has been used.

Now suppose that the constant $c \neq 0$. Then (7.4) shows that one may use the translational freedom in the coordinate $u$ to arrange that $\rho=c u$. If $c=0$ then the first equation in (7.5) together with the non-vanishing of $p_{a ; b}$ on $V$ implies $\rho=0$ on $V$ and the second equation in (7.4) shows that $\sigma$ is a non-zero constant on $V$. Then (7.7) and (7.8) reveal that $\delta$ is a linear function of $u$ which is nowhere zero on $V$. A translation of the $u$ coordinate then ensures that $\delta=\sigma u$ (and $u$ is nowhere zero) on $V$. Thus whether $c$ is zero or not, one can achieve the result that $u p_{a ; b}=T_{a b}$. Denoting the Christoffel symbols arising from $\nabla$ by $\Gamma$ and noting that since $l=\partial / \partial v$ is a Killing vector field on $V$, the functions $g_{\alpha \beta}\left(=T_{\alpha \beta}\right)$ are independent of $v$, one calculates that $-u \Gamma_{\alpha \beta}^{2}=g_{\alpha \beta}$. This, together with $u p_{a ; b}=T_{a b}$ and an integration then leads to $g_{\alpha \beta}=u^{2} h_{\alpha \beta}$ for functions $h_{\alpha \beta}$ which are independent of $u$ and $v$. Thus, on $V$, the original metric is

$$
d s^{2}=2 d u d v+u^{2} h_{\alpha \beta}\left(x^{3}, x^{4}\right) d x^{\alpha} d x^{\beta} .
$$

The next step is, recalling the coordinate scalings above, to write $\rho=c u$ and, from (7.4), $\sigma=c v+e_{1}$ and substitute into (7.7) to get $\beta=c v^{2}+2 e_{1} v+e_{2}, \gamma=c u^{2}+e_{3}$ and $\delta=e_{1} u+c u v+e_{4}$ for constants $e_{1}, e_{2}, e_{3}$ and $e_{4}$. Then (7.5) and (7.8) act as consistency checks on these constants and give $e_{3}=e_{4}=0$. The equation (7.7) now gives the Sinyukov tensor in (7.3) as

$$
a_{a b}=\phi\left(g_{a b}+\left(c v^{2}+2 e_{1} v+e_{2}\right) l_{a} l_{b}+c u^{2} p_{a} p_{b}+\left(e_{1} u+c u v\right)\left(l_{a} p_{b}+p_{a} l_{b}\right)\right) .
$$

This equation can then be inverted following the techniques described in Section 5 . First, with the above values for $\rho$ and $\sigma$ one finds $\lambda$ from (7.1) and, from (7.11), $\psi$ can be calculated from the equation $\psi_{a}=-a_{a b}^{-1} \lambda^{b}$ given in Section 5. Then the potential, $\chi$, of the 1-form $\psi$ can be found. Finally the required metric is then given, on $V$, by $g_{a b}^{\prime}=e^{2 \chi} a_{a b}$. This calculation was done using Maple and results in

$$
\psi=d \chi, \quad \chi=\frac{1}{2} \ln F, \quad F=\kappa^{4}\left[1+2 c u v+2 e_{1} u+\left(e_{1}^{2}-c e_{2}\right) u^{2}\right]^{-1}
$$


and for the metric $g^{\prime}$, projectively related to $g$,

$$
\begin{aligned}
d s^{\prime 2}= & \kappa F g-\kappa^{-3} F^{2}\left[\left(c v^{2}+2 e_{1} v+e_{2}\right) d u^{2}+c u^{2} d v^{2}\right. \\
& \left.+2 u\left(c v+e_{1}+\left(e_{1}^{2}-c e_{2}\right) u\right) d u d v\right]
\end{aligned}
$$

for a positive constant $\kappa=\phi^{-1}$.

It is noted here that if $\lambda_{a}(m)$ is proportional to $l(m)$ then it follows from $(7.1)$ that $\rho(m)=0$ and from (7.5) that $c=0$. Then (7.4) reveals that $\rho=0$ and that $\sigma$ is constant on $V$ and so $\lambda_{a}$ is a constant multiple of $l_{a}=g_{a b} l^{b}$ on $V$. Conversely, since $\nabla \lambda=c g$, this last condition implies that $c=0$. Thus the special case $c=0$ corresponds to the condition that $\lambda_{a}$ is a (non-zero) constant multiple of $l_{a}$ on $V$ and then (7.11), (7.12) and (7.13) simplify. For this special case it follows from (7.12) that $\psi_{a}=r(u) l_{a}$ on $V$ for some (smooth) function $r$ on $V$. Thus, for any metric $g^{\prime}$, projectively related to $g$ on $V$ and with Levi-Civita connection $\nabla^{\prime}$, one finds from (5.1) that $l_{a \mid b}=-2 r(u) l_{a} l_{b}$, where a stroke denotes a $\nabla^{\prime}$-covariant derivative. Then the nowhere-zero vector field $l^{\prime} \equiv e^{\int 2 r} l$ on $V$ is null with respect to $g^{\prime}$ (from (7.13) with $c=0$ ) and covariantly constant with respect to $\nabla^{\prime}$. It is, in fact, true that $\left(V, g^{\prime}\right)$ (that is, $\left.\nabla^{\prime}\right)$ is also of holonomy type $R_{11}$. This follows from Table 1 since $\left(V, g^{\prime}\right)$ admits a nowhere zero null covariantly constant vector field and so must be of holonomy type $R_{3}, R_{4}, R_{8}$ or $R_{11}$ and the first three of these are ruled out because they would, from Theorems 6.1 and 6.4 , force $\nabla=\nabla^{\prime}$. [That $\nabla^{\prime}$ cannot be flat over some non-empty open subset $V^{\prime}$ of $V$ follows since $g^{\prime}$ would then be of constant curvature and hence so would $g$ [16], contradicting the curvature class $\mathbf{D}$ condition on $(V, g)$.] The space-time $\left(V, g^{\prime}\right)$ is also of curvature class $\mathbf{D}$ on $V$ since, otherwise, it would be of curvature class $\mathbf{C}$ over some non-empty open subset $V^{\prime}$ of $V$. In this case, Theorem 6.3 would show that $\lambda=0$ on $V^{\prime}$ and a contradiction follows.

Now let $(M, g)$ be a space-time of holonomy type $R_{10}$ or $R_{13}$ which is (non-flat and) of curvature class $\mathbf{D}$, that is the curvature rank equals 1 over some open dense subset $U$ of $M$ and Riem vanishes on $M \backslash U$. Let $g^{\prime}$ be a metric on some connected open neighbourhood $V \subset U$ of $m \in U$ which is projectively related to $g$ on $V$. On $V$, Riem takes the form (3.3) for some nowhere zero bivector $F$ on $V$. If the holonomy type is $R_{13}$ this bivector $F$ is necessarily spacelike and so the subset $\mathbf{D}_{\mathbf{n}}$ in the above decomposition of $M$ is empty. Then $V$ may be chosen to admit a covariantly constant, unit timelike vector field $u$ (so that, from the Ricci identity, $R_{b c d}^{a} u^{d}=0$ on $V$ ) and a unit (smooth) spacelike vector field $w$ which is orthogonal to $u$ on $V$ and also satisfies $R_{b c d}^{a} w^{d}=0$ on $V$. On $V$ one has the useful relations $u^{a} u_{a ; b}=w^{a} w_{a ; b}=u^{a} w_{a ; b}=0$. If, however, the holonomy type of $(M, g)$ is $R_{10}, V$ may be chosen so as to admit a covariantly constant unit spacelike vector field (which for later convenience will also be labelled $u$ ) and which satisfies $R_{b c d}^{a} u^{d}=0$ and another nowhere-zero vector field (similarly labelled $w$ ) which is orthogonal to $u$ and satisfies $R_{b c d}^{a} w^{d}=0$ on $V$ but whose nature (spacelike, timelike or null) may vary. It follows that in the $R_{10}$ case, each of $\mathbf{D}_{\mathbf{n}}$ and $\mathbf{D}_{\mathbf{n n}}$ may be non-empty. These remarks permit the totality of possibilities for the types $R_{10}$ and $R_{13}$, with one exception, to be taken together (the exceptional case being for type $R_{10}$ and the subset $\mathbf{D}_{\mathbf{n}}$ ).

The proof is, in fact, very similar to that already given for the holonomy type $R_{11}$ and so it will be described only briefly. For $m \in U$ choose an open, connected and simply connected neighbourhood $V$ of $m$. Then one has, from Lemma 5.1, $\lambda_{a ; b}=c g_{a b}$ for some constant $c$ and so, as before,

$$
\lambda_{a}=\sigma u_{a}+\rho w_{a}, \quad \rho w_{a ; b}+w_{a} \rho_{, b}+u_{a} \sigma_{, b}=c g_{a b}
$$

and

$$
a_{a b}=\phi g_{a b}+\beta u_{a} u_{b}+\gamma w_{a} w_{b}+\delta\left(u_{a} w_{b}+w_{a} u_{b}\right)
$$


for functions $\sigma, \rho, \phi, \beta, \gamma$ and $\delta$ on $V$. Whichever subcase is chosen an argument similar to that given in the holonomy $R_{11}$ case leads to the conclusion that $\phi$ is constant.

Now suppose the holonomy type is $R_{10}$, that int $\left(M_{2} \cap \mathbf{D}_{\mathbf{n}}\right)$ is non-empty and with $V$ as usual choose $m \in V \subset \operatorname{int}\left(M_{2} \cap \mathbf{D}_{\mathbf{n}}\right)$. On $V, u$ is unit spacelike and covariantly constant and $w$ is null. Then the second of (7.14), on contraction with $w^{a}$ and $u^{a}$, gives $c=0, \sigma$ is constant on $V$ and $(\rho w)_{a ; b}=0$. Thus $\lambda$ is covariantly constant on $V$ and $\rho(m)=0$. [If $\rho(m) \neq 0$ then $\rho$ would not vanish over some neighbourhood of $m$ and on that neighbourhood $\rho w$ would be a nowhere-zero, nowhere-null covariantly constant vector field on $V$ with $\rho w(m)$ and $u(m)$ independent members of $T_{m} M$ at each $m \in V$. This would reduce the holonomy type in this neighbourhood to $R_{3}$ and then, from Theorem 6.1, one finds that $\lambda$ vanishes on this neighbourhood of $m$ contradicting the fact that $m \in M_{2}$.] Since $\lambda$ (and $u$ ) are covariantly constant on $V$ with $\lambda$ proportional to $u$ at $m$ it follows that $\lambda$ is proportional to $u$ on $V$ and hence, from (7.14), that $\rho$ is zero on $V$. Finally a substitution of (7.15) into (5.7) and a contraction with $u^{a} w^{b}$ gives $\sigma=0$ on $V$ and hence, from (7.14) that $\lambda \equiv 0$ on $V$. This contradiction to the statement $m \in M_{2}$ shows that $\operatorname{int}\left(M_{2} \cap \mathbf{D}_{\mathbf{n}}\right)=\varnothing$.

The other cases can be handled together. One assumes that $M_{2} \cap \mathbf{D}_{\text {nn }}$ is non-empty and chooses $m \in\left(M_{2} \cap \mathbf{D}_{\mathbf{n n}}\right)$ and the usual neighbourhood $V$ such that $m \in V \subset \mathbf{D}_{\mathbf{n n}}$. To deal with both cases simultaneously one writes, for the vector fields $u$ and $w$ on $V, u^{a} u_{a}=\epsilon_{1}, w^{a} w_{a}=\epsilon_{2}$ (and $u^{a} w_{a}=0$ ) with $\epsilon_{1}$ and $\epsilon_{2}$ equal to either \pm 1 . Thus the $R_{13}$ type requires $\epsilon_{1}=-1$ and $\epsilon_{2}=1$ whilst the $R_{10}$ type requires $\epsilon_{1}=1$ and $\epsilon_{2}= \pm 1$. Equations (7.14) and (7.15) still hold and contractions of the second in (7.14) with $u^{a}$ and $w^{a}$ give

$$
\epsilon_{1} \sigma_{, a}=c u_{a}, \quad \epsilon_{2} \rho_{, a}=c w_{a}, \quad \rho w_{a ; b}=c T_{a b} \quad\left(T_{a b}=g_{a b}-\epsilon_{1} u_{a} u_{b}-\epsilon_{2} w_{a} w_{b}\right) .
$$

Then substituting (7.15) into (5.7) and contracting successively with $u^{a} u^{b}, w^{a} w^{b}$ and $u^{a} w^{b}$ and then with $u^{a}$ and $w^{a}$ gives

$$
2 \epsilon_{1} \sigma u_{a}=\beta_{, a}, \quad 2 \epsilon_{2} \rho w_{a}=\gamma_{, a}, \quad \epsilon_{2} \rho u_{a}+\epsilon_{1} \sigma w_{a}=\epsilon_{1} \epsilon_{2} \delta_{, a}
$$

and

$$
\gamma w_{a ; b}=\rho T_{a b}, \quad \delta w_{a ; b}=\sigma T_{a b} .
$$

As before one can now show, since $\lambda \in M_{2}$, that $\lambda$ and $w_{a ; b}$ are nowhere zero on $V$ and hence $V \subset M_{2} \cap \mathbf{D}_{\mathbf{n n}}$ (so that $M_{2} \cap \mathbf{D}_{\mathbf{n n}}$ is open in $M$ ). Also, on $V, w_{a ; b}$ is a non-zero multiple of $T_{a b}$. Thus, $V$ may be chosen so that the 1-forms associated with $u$ and $w$ through $g$ are global gradients on $V$, say $u=\epsilon_{1} d t$ and $w=\epsilon_{2} d z$ for functions $t$ and $z$ on $V$. So reducing $V$, if necessary, to a coordinate domain with coordinates $t, z, x^{3}, x^{4}$ such that $g$ takes the form

$$
d s^{2}=\epsilon_{1} d t^{2}+\epsilon_{2} d z^{2}+z^{2} h_{\alpha \beta}\left(x^{3}, x^{4}\right) d x^{\alpha} d x^{\beta},
$$

where the functions $h_{\alpha \beta}$ are independent of $t$ and $z$. The equations (7.14) and (7.17) may be integrated to give

$$
a_{a b}=\phi\left(g_{a b}+\left(c t^{2}+2 c_{2} t+c_{3}\right) u_{a} u_{b}+c z^{2} w_{a} w_{b}+\left(c t z+c_{2} z\right)\left(u_{a} w_{b}+w_{a} u_{b}\right)\right),
$$

where $c_{2}$ and $c_{3}$ are constants. Inverting the pair $(a, \lambda)$ to obtain the corresponding pair $\left(g^{\prime}, \psi\right)$ leads to

$$
\begin{aligned}
& \psi=d \chi, \quad \chi=\frac{1}{2} \ln F \\
& F=\kappa^{4}\left[1+\epsilon_{2}\left(c+\epsilon_{1}\left(c_{3} c-c_{2}^{2}\right)\right) z^{2}+\epsilon_{1}\left(c t^{2}+2 c_{2} t+c_{3}\right)\right]^{-1}
\end{aligned}
$$


corresponding to the metric $g^{\prime}$ projectively related to $g$ and given by

$$
g^{\prime}=\kappa F g-\kappa^{-3} F^{2}\left(\begin{array}{l}
\left(c t^{2}+2 c_{2} t+c_{3}+\epsilon_{2}\left(c c_{3}-c_{2}^{2}\right) z^{2}\right) d t^{2} \\
+\left(c+\epsilon_{1}\left(c c_{3}-c_{2}^{2}\right)\right) z^{2} d z^{2}+2 \epsilon_{1} \epsilon_{2}\left(c t+c_{2}\right) z d t d z
\end{array}\right)
$$

where $\kappa=\phi^{-1}$ is a positive constant.

In summary, for space-times $(M, g)$ of holonomy types $R_{10}, R_{11}$ and $R_{13}$ and which are of curvature class $\mathbf{D}$ over some open dense subset $U$ of $M$, $\operatorname{int}\left(M_{2} \cap \mathbf{D}_{\mathbf{n}}\right)=\varnothing$ and one may identify an open dense subset of $M$ such that each point $m$ of this subset lies in a neighbourhood where either $\lambda=0$ (when $m \in M_{1}$ ) or where $\lambda$ is nowhere zero and in which the situation can be completely resolved (in the open subset $M_{2} \cap \mathbf{D}_{\mathbf{n n}}$ ). It is interesting to remark at this point that more can be said in the cases when Riem is nowhere zero on $M$ and the nature (timelike, spacelike or null) of the bivector $F$ in (3.3) is constant on $M$. To see this, suppose that such is the case and suppose that $F$ is non-null (and hence always timelike or spacelike on $M$ ). Then, in the previous notation, $M=M_{1} \cup M_{2}=\mathbf{D}_{\text {nn }}$ with $M_{1}$ open in $M$. But then the previous work shows that $M_{2}=M_{2} \cap \mathbf{D}_{\text {nn }}$ is also open in $M$. Thus, since $M$ is connected, one of $M_{1}$ and $M_{2}$ is empty and so either $M=M_{1}$ or $M=M_{2}$. It follows that, in this case, either $\lambda=0$ on $M$ or $\lambda$ is nowhere vanishing on $M$. In the similar situation, but with $F$ null, $M=\mathbf{D}_{\mathbf{n}}$ and the work above shows that $\lambda=0$ on $M$.

Although the class $\mathbf{D}$ assumption for these holonomy types was made over an open dense subset of $M$ it is now seen that this is, in fact, a complete solution for holonomy types $R_{10}, R_{11}$ and $R_{13}$. For if $(M, g)$ is of any of these holonomy types, then either it is of curvature class $\mathbf{C}$ over an open dense subset of $M$, in which case, $\nabla=\nabla^{\prime}$ on $M$, or the curvature class is $\mathbf{D}$ over some subset $E$ of $M$. Now the subset $J$ of $M$ over which Riem vanishes is closed and satisfies int $J=\varnothing$. Also, the subset $E \cup J$ is closed (by the rank theorem on Riem since $M \backslash(E \cup J)$ is exactly the open subset of $M$ on which Riem has curvature rank $>1)$ and if int $E=\varnothing$ it is easily checked that int $(E \cup J)=\varnothing$ and so the curvature class is $\mathbf{C}$ over the open dense subset $M \backslash(E \cup J)$ of $M$. Then Theorem 6.3 gives $\nabla=\nabla^{\prime}$ on $M$. If $\operatorname{int} E \neq \varnothing$ let $E^{\prime}=\operatorname{int} E$ and proceed as above on the (connected) components of $E^{\prime}$ with metric $g$.

Where a space-time $(M, g)$ has holonomy type $R_{9}$ or $R_{14}$ the situation turns out to be even more complicated. These cases will be discussed in detail elsewhere [25], but, for completeness, non-trivial (that is, $\lambda$ not identically zero) examples of projective relatedness in such space-times will now be discussed briefly.

Consider a spacetime $(M, g)$ and a point $m \in M$ for which there exists a coordinate neighbourhood $U$ of $m$ with coordinates $u, v, x, y(v>0)$ such that $g$ is given on $U$ by

$$
d s^{2}=2 d u d v+b(u) \sqrt{v} d u^{2}+u^{2} e^{f(x, y)}\left(d x^{2}+d y^{2}\right),
$$

where $b(u)$ and $f(x, y)$ are arbitrary smooth functions on $U$. Now the 1-form field $l=d u$ may be shown to be recurrent on $U$, and is covariantly constant on $U$ if and only if $b(u) \equiv 0$ on $U$. On any connected open subset of $U$ where $b(u)=0,(7.23)$ is locally of the form (7.10) and of curvature class $\mathbf{D}$. However, for (7.23) to be of holonomy type $R_{9}$ or $R_{14}, b(u) \neq 0$ at least at one point $m \in M$, and hence over some open neighbourhood $V$ of $m$. Assuming that $b(u)$ is nowhere zero on $V$ the curvature class is $\mathbf{A}$ on $V$ and after somewhat lengthy calculations it may be shown that (5.7) may be solved non-trivially to give

$$
a=\phi\left(g+2 \xi u d u d v+\xi(2 v+u b(u) \sqrt{v}) d u^{2}\right)
$$

where $\phi, \xi \in \mathbb{R}$. Inverting the pair $(a, \lambda)$ to obtain the corresponding pair $\left(g^{\prime}, \psi\right)$ leads to

$$
\psi=d \chi, \quad \chi=\frac{1}{2} \ln F, \quad F=\kappa^{4}[1+\xi u]^{-2}
$$


with $\kappa=\phi^{-1}$, corresponding to a metric $g^{\prime}$, projectively related to (7.23), and given on $V$ by

$$
g^{\prime}=\kappa F g-\kappa^{-3} F^{2}\left[2 \xi u(1+\xi u) d u d v+\xi(u(1+\xi u) b(u) \sqrt{v}+2 v) d u^{2}\right] .
$$

If $f(x, y)$ satisfies $\frac{\partial^{2} f}{\partial x^{2}}+\frac{\partial^{2} f}{\partial y^{2}}=0$ everywhere on $V$ then an application of the Ambrose-Singer theorem [14] shows (7.23) has holonomy type $R_{9}$, and otherwise has holonomy type $R_{14}$ on $V$.

It is remarked that examples are known of non-trivially projectively related space-times of the Friedmann-Robertson-Walker-Lemaitre (FRWL) type and which are of holonomy type $R_{15}$ [26] . Other examples of $R_{15}$ metrics with similar properties will be discussed in [25].

\section{Conclusions}

This paper studied the question of, given a space-time $(M, g)$, what can be said about those other metrics $g^{\prime}$ on $M$ (or some open submanifold of $M$ ) which are projectively related to $g$ ? The question was inspired from both a geometrical point of view and from a physical standpoint through the principle of equivalence in Einsteins general theory of relativity. If $(M, g)$ is an Einstein space (and this includes the important vacuum metrics of general relativity) the problem is already resolved, as explained in Section 5. This paper then proceeded to extend this by studying the situation through the various possible holonomy groups that are possible for a Lorentz manifold. It was shown that, by using certain algebraic properties of the curvature tensor, much of this problem is tractable and an essentially complete solution was obtained for all the holonomy types except $R_{9}, R_{14}$ and $R_{15}$. For these latter types, examples of non-trivially projectively related space-times were shown to exist (and further details will be given elsewhere). Amongst all of these are several well-known, physically important solutions of Einsteins field equations.

\section{References}

[1] Geroch R.P., Spinor structure of space-times in general relativity, J. Math. Phys. 9 (1968), 1739-1744.

[2] Hall G.S., Symmetries and curvature structure in general relativity, World Scientific Lecture Notes in Physics, Vol. 46, World Scientific Publishing Co., Inc., River Edge, NJ, 2004.

[3] Hall G.S., McIntosh C.G.B., Algebraic determination of the metric from the curvature in general relativity, Internat. J. Theoret. Phys. 22 (1983), 469-476.

[4] Hall G.S., Curvature collineations and the determination of the metric from the curvature in general relativity, Gen. Relativity Gravitation 15 (1983), 581-589.

[5] McIntosh C.G.B., Halford W.D., Determination of the metric tensor from components of the Riemann tensor, J. Phys. A: Math. Gen. 14 (1981), 2331-2338.

[6] Hall G.S., Lonie D.P., On the compatibility of Lorentz metrics with linear connections on four-dimensional manifolds, J. Phys. A: Math. Gen. 39 (2006), 2995-3010, gr-qc/0509067.

[7] Kobayashi S., Nomizu K., Foundations of differential geometry, Vol. 1, Interscience Publishers, New York, 1963.

[8] Schell J.F., Classification of four-dimensional Riemannian spaces, J. Math. Phys. 2 (1961), 202-206.

[9] Hall G.S., Lonie D.P., Holonomy groups and spacetimes, Classical Quantum Gravity 17 (2000), 1369-1382, gr-qc/0310076.

[10] Hall G.S., Covariantly constant tensors and holonomy structure in general relativity, J. Math. Phys. 32 (1991), 181-187.

[11] Besse A., Einstein manifolds, Springer-Verlag, Berlin, 1987.

[12] Wu H., On the de Rham decomposition theorem, Illinois J. Math. 8 (1964), 291-311.

[13] Hall G.S., Kay W., Holonomy groups in general relativity, J. Math. Phys. 29 (1988), 428-432.

[14] Ambrose W., Singer I.M., A theorem on holonomy, Trans. Amer. Math. Soc. 75 (1953), 428-443. 
[15] Hall G.S., Connections and symmetries in space-times, Gen. Relativity Gravitation 20 (1988), 399-406.

[16] Eisenhart L.P., Riemannian geometry, Princeton University Press, Princeton, 1966.

[17] Thomas T.Y., Differential invariants of generalised spaces, Cambridge, 1934.

[18] Weyl H., Zur Infinitesimalgeometrie: Einordnung der projectiven und der konformen Auffassung, Gött. Nachr. (1921), 99-112.

[19] Petrov A.Z., Einstein spaces, Pergamon Press, Oxford - Edinburgh -New York, 1969.

[20] Sinyukov N.S., Geodesic mappings of Riemannian spaces, Nauka, Moscow, 1979 (in Russian).

[21] Mikes J., Hinterleitner I., Kiosak V.A., On the theory of geodesic mappings of Einstein spaces and their generalizations, in The Albert Einstein Centenary International Conference, Editors J.-M. Alini and A. Fuzfa, AIP Conf. Proc., Vol. 861, American Institute of Physics, 2006, 428-435.

[22] Hall G.S., Lonie D.P., The principle of equivalence and projective structure in spacetimes, Classical Quantum Gravity 24 (2007), 3617-3636, gr-qc/0703104.

[23] Kiosak V., Matveev V.A., Complete Einstein metrics are geodesically rigid, Comm. Math. Phys. 289 (2009), 383-400, arXiv:0806.3169.

[24] Hall G.S., Lonie D.P., Projective equivalence of Einstein spaces in general relativity, Classical Quantum Gravity 26 (2009), 125009, 10 pages.

[25] Hall G.S., Lonie D.P., Holonomy and projective structure in space-times, Preprint, University of Aberdeen, 2009.

[26] Hall G.S., Lonie D.P., The principle of equivalence and cosmological metrics, J. Math. Phys. 49 (2008), 022502, 13 pages. 